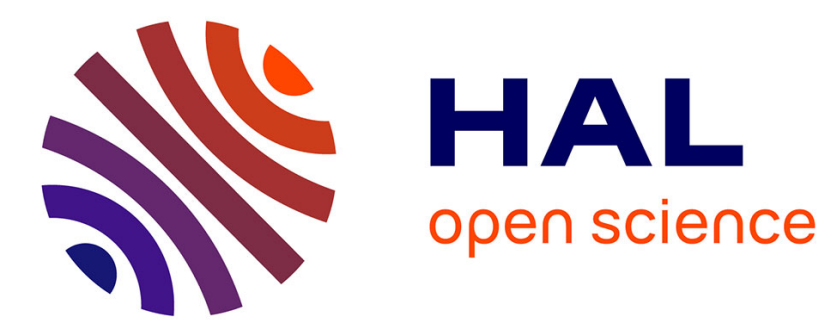

\title{
On Wind-Driven Energetics of Subtropical Gyres
}

\author{
Q. Jamet, B. Deremble, N. Wienders, T. Uchida, W. Dewar
}

\section{To cite this version:}

Q. Jamet, B. Deremble, N. Wienders, T. Uchida, W. Dewar. On Wind-Driven Energetics of Subtropical Gyres. Journal of Advances in Modeling Earth Systems, 2021, 13 (4), 10.1029/2020MS002329 . hal-03501807

\section{HAL Id: hal-03501807 \\ https://hal.science/hal-03501807}

Submitted on 24 Dec 2021

HAL is a multi-disciplinary open access archive for the deposit and dissemination of scientific research documents, whether they are published or not. The documents may come from teaching and research institutions in France or abroad, or from public or private research centers.
L'archive ouverte pluridisciplinaire HAL, est destinée au dépôt et à la diffusion de documents scientifiques de niveau recherche, publiés ou non, émanant des établissements d'enseignement et de recherche français ou étrangers, des laboratoires publics ou privés.

\section{(c)(1)}

Distributed under a Creative Commons Attribution| 4.0 International License 


\section{AMES Journal of Advances in Modeling Earth Systems}

\author{
RESEARCH ARTICLE \\ 10.1029/2020MS002329 \\ Key Points: \\ - Multiple-scale analysis of the \\ wind-driven circulation argues the \\ primary sink of energy occurs in the \\ separated boundary current jet \\ - Analyses of a $1 / 12^{\circ}$ ocean \\ simulation supports the theory; \\ coastal topographic features play a \\ secondary role \\ - Subtropical gyre dynamics are \\ largely inertial, recirculating large \\ amount of energy in comparison to \\ wind energy input
}

Correspondence to:

W. K. Dewar,

wdewar@fsu.edu

Citation:

Jamet, Q., Deremble, B., Wienders, N., Uchida, T., \& Dewar, W. K. (2021). On wind-driven energetics of subtropical gyres. Journal of Advances in Modeling Earth Systems, 13, e2020MS002329. https://doi.org/10.1029/2020MS002329

Received 3 SEP 2020 Accepted 14 JAN 2021
C 2021. The Authors

This is an open access article under the terms of the Creative Commons Attribution License, which permits use, distribution and reproduction in any medium, provided the original work is properly cited.

\section{On Wind-Driven Energetics of Subtropical Gyres}

\author{
Q. Jamet ${ }^{1}$, B. Deremble ${ }^{1}$, N. Wienders ${ }^{2}$ (D), T. Uchida ${ }^{1}$ (i), and W. K. Dewar ${ }^{1,2}$ (i) \\ ${ }^{1}$ CNRS, IRD, Grenoble INP, IGE, Université Grenoble Alpes, Grenoble, France, ${ }^{2}$ Department of EOAS, Florida State \\ University, Tallahassee, FL, USA
}

\begin{abstract}
The flow of energy in the wind-driven circulation is examined in a combined theoretical and numerical study. Based on a multiple-scale analysis, we find the mesoscale field in the ocean interior is strongly affected by, but does not feed back onto, the ventilated thermocline. In the western boundary region, the associated currents first appear as coastal jets, conserving mean energy, and later as separated jet extensions where the mesoscale is energized by the mean field. It is in the separated jet zone where the primary loss of general circulation energy to the mesoscale occurs. These ideas are tested by an analysis of a regional $1 / 12^{\circ}$ primitive equation numerical model of the North Atlantic. The predictions of the theory are generally supported by the numerical results. The one exception is that topographic irregularities in the coastal jet spawn eddies, although these eddies contribute modestly to the energy budget. We therefore conclude the primary sink of wind input into the mean circulation is in the separated jet, and not the interior. The analysis also shows wind energy input to be much smaller than the interior energy fluxes; thus, the general circulation largely recirculates energy.

Plain Language Summary Atmospheric winds provide energy to the ocean general circulation through surface stress, forcing the so-called wind-driven oceanic gyres. Although the primary energy sink of this large-scale circulation is usually recognized to be energy transfers toward smaller scales, details remain unclear. In this paper, we argue that the ocean receives energy over the broader interior from the wind and recirculates that energy to the open ocean Gulf Stream, where it is lost to ocean eddies. We test these ideas by analyzing a $1 / 12^{\circ}$, primitive equations numerical simulation of the North Atlantic. The predictions of the theory are generally supported by the numerical results. Lastly, we note the energy moved in the general circulation greatly exceeds that added by the wind, implying the circulation acts like a flywheel. This very inertial character of the circulation resembles a classical model first recognized by Fofonoff in 1954.
\end{abstract}

\section{Introduction}

Ocean energy balance have received considerable recent attention, motivated in part by interest in ocean mixing. As discussed by Wunsch and Ferrari (2004) and Ferrari and Wunsch (2009), maintenance of ocean stratification requires mixing that, in turn, requires energy. The rates of energy consumption by mixing consistent with the observed ocean stratification are thought to be $1-2$ Terawatts (TW; $1 \mathrm{TW}=10^{12} \mathrm{~W}$, St. Laurent \& Simmons, 2006). High-frequency winds and tides can provide such power (e.g., Ferrari \& Wunsch, 2009), and both are broadly accepted as being principally involved in ocean mixing.

Low-frequency winds globally also provide energy ( $\leq 1 \mathrm{TW}$ ) to the ocean (Wunsch, 1998; Zhai et al., 2012), primarily driving large-scale horizontal circulations in the process. The input is divided geographically between the Southern Ocean, recipient of about $60 \%$ of the total, the equatorial Pacific and the subtropical gyres. A prevailing idea is that the large-scale circulation loses its energy to the mesoscale by means of geostrophic instabilities, with the associated flux to the mesoscale being roughly 1 TW (Ferrari \& Wunsch, 2009; Wunsch \& Ferrari, 2004). The mean flow equilibration then consists of wind energy input balanced by loss to the mesoscale. The primary focus of this study is the subtropical gyres component of the overall energy story. Specifically, we examine how and where the wind-driven subtropical gyres achieve their equilibrium.

The role of the mesoscale in circulation dynamics has been a focus of numerical simulations since the studies of Holland and Lin (1975), Semtner and Mintz (1977), Robinson et al. (1977), and Holland (1978). Working in few layer, primitive equation and quasigeostrophic (QG) environments, these early studies related the appearance of eddies to large-scale flow instabilities, and documented their role in developing deep 
mean circulations. The role of eddy energy was explored in the work by Harrison and Robinson (1978), where the importance of the recirculation to eddy development was noted. The paper by Holland (1978) provides a thorough discussion of basin integrated energy budgets in a QG setting. More recently, the rich, detailed structure and exchange of energy between mean and eddy flows have been illustrated in primitive equation based North Atlantic simulations (Greatbatch et al., 2010). The paper by Zhai and Marshall (2013) focuses on vertical eddy pressure work in the North Atlantic, arguing that it plays a major role in balancing mean gyre wind forcing.

The effect of the mesoscale on the large-scale circulation has also been addressed in situ. An early examination is the classic paper by Gill et al. (1974), who used a combination of theory and observations to suggest that the interior westward mean flow of the North Atlantic subtropical gyre is an important site for eddy development. The authors argued that the net release from the mean potential energy field to eddies via baroclinic instability appeared to be adequate to balance the net input of energy from the mean wind. These estimates were based on local, linearized QG theory.

The present study departs from past efforts by a combination of theoretical and numerical examination which seeks to identify how the subtropical wind-driven circulation obtains a leading-order energy balance. We first provide a short review of past efforts for coupling planetary geostrophic and QG equations leading the main motivations for the present study (Section 2.) A classical multiple-scale analysis of the ocean interior is then applied (Section 3 and Appendix A), and argues the local effects of eddies are relatively weak. Augmenting the analysis with an anisotropic western boundary layer suggests the western boundary current, along the ocean boundary, is also only weakly effected by the mesoscale. This leaves the open ocean extension of the Gulf Stream as primary location where the mean flow drains energy to the mesoscale. A North Atlantic numerical simulation is analyzed in Section 4 from the perspective of the theory and found to support it. It is in the desire to interpret large-scale circulation theory in terms of variable regional energy exchange with the mesoscale that this paper differs from previous examination of regional Gulf Stream energy budgets. A more classical, recent quantification of regional exchanges and connections to Lorenz energy cycles can be found in the study of Kang and Curchitser (2015). The paper concludes with a Summary and a discussion of potential future directions (Section 5).

\section{Background}

Pedlosky (1984, P84 hereafter), in an insightful contribution, attempted a synthesis of the two primary models of the wind-driven ocean general circulation, that is, the ventilated thermocline (Luyten et al., 1983), framed in the "Planetary Geostrophic" (PG hereafter) equations, and homogenization theory (Rhines \& Young, 1982), which employs the "QG" equations. The scaling behind PG implies it is meant to describe basin scale motions (thousands of kilometers), while the QG equation applies to smaller scale motions, typically of the order of the Rossby deformation radius (several tens of kilometers). P84 performed the synthesis using a multiple-scale approach and found that the QG field was strongly affected at leading order by the PG field, but that no comparable feedback onto the PG field from the QG field occurred (see also Grooms et al., 2011). Only at higher order it is possible to locate feedbacks from the QG field onto the PG field.

The full implications of this result have not received much attention, particularly that the forced PG field, in its lack of interaction with the QG eddy field, does not have an obvious energy sink and thus suffers from an unbalanced energy budget. While it is possible that one or both of the ventilated thermocline or QG theory are flawed, both have a considerable literature behind them supporting their quantitative and qualitative utility.

A different possibility explored here is that the multiple-scale QG and ventilated thermocline theory is correct, but incomplete. This idea was first explored in Grooms et al. (2011) who elaborated on P84 by allowing for anisotropy. This extends P84 by recognizing that QG and PG are fundamentally zonally and meridionally isotropic in their scaling assumptions. In contrast, features like the Gulf Stream and the Kuroshio are not well described within either framework. Both are arguably features midway between PG and QG, characterized by the deformation radius in the cross-jet direction and the planetary scale in the along-jet direction. Grooms et al. (2011) show how involving anisotropy leads to equations where planetary scale and deformation radius scale motions can interact at leading order. 
As a consequence, jets might be regions in which large-scale energy is extracted from the flow. Our present analysis supports this idea; however, it does so by emphasizing the role of the separated jet, and not the western intensified flow along the coast. This is implicit in some previous work, for example, the maps of vertical pressure work in Zhai and Marshall (2013), the potential vorticity study of Deremble et al. (2014), and the momentum budgets proposed in the studies by Hughes and de Cuevas (2001) and Schoonover et al. (2016). We will capitalize on these results when developing an anisotropic boundary layer model to append to the ventilated thermocline interior. We depart from Grooms et al. (2011) by arguing that the coastal components of western boundary layers are only weakly eddying and focus on their open ocean extensions. These regions lie outside of both QG and PG regimes, although they involve mesoscale flow at leading order. It also emerges, from a theoretical point of view, that the separated jet appears as the most important region where the mesoscale grows at the expense of the mean state.

\section{General Circulation Structure and Energy}

We develop here a theoretical framework later analyzed in Section 4 by the means of quantitative analysis of the flow of energy in a North Atlantic simulation. Specifically, the circulation is divided into subregions, each possessing a distinct dynamical character. The leading-order statements of energy balance in each region reflect the regional dynamics, which in turn provides a roadmap for examining the numerical results.

\subsection{Regional Dynamics}

As in P84, we analyze the hydrostatic, primitive equations of motion via a multiple-scales approach capitalizing on the space and time scale separation between PG and QG dynamics. The analysis itself is somewhat lengthy; we therefore present the details in Appendix A and quote the primary results here.

The first result is that the ocean interior is governed by the well-known theory of the ventilated thermocline, that is, the flow is PG, wind-forced and conservative of large-scale potential vorticity whose structure is set at outcrops. What the analysis adds to the classical demonstration of Luyten et al. (1983) is that the ventilated thermocline sets the environment housing a QG eddy field. In contrast, the QG field does not feed back on the ventilated thermocline. This is a result first seen in P84, and subsequently in Grooms et al. (2011), and so is not new, per se. What is new here is our interpretation of this result, which is that the ventilated thermocline is not energetically closed, that is, the flow is forced but not damped. One must look elsewhere to determine how the circulation equilibrates.

An obvious location to expect energy loss is the western boundary layer, as it plays that role in classical general circulation theories. We probe this possibility through allowing for anisotropy in the analysis, following an approach first introduced in Grooms et al. (2011). Our approach and theirs differ in detail, and end up with different boundary layer structures as encapsulated in reduced equation sets. We argue the coastal Gulf Stream, constrained as it is by topography, is essentially an inertial boundary layer. It accepts a mass influx from the interior ventilated thermocline, and then simply redirects the flux along the coast to the point of separation. The principle time scales of the boundary jet are set by the interior, implying the mean flow does not lose energy to the rapid mesoscale. This differs from the study by Grooms et al. (2011) who invoked a Gent-McWilliams parameterization in the boundary layer to model mean flow to mesoscale transfers. Thus, we conclude that the coastal Gulf Stream does not act as a sink of general circulation energy and that energetic equilibration of the circulation occurs elsewhere.

Beyond the coastal Gulf Stream lies the separated jet region, which consists of a jet-like coherent flow extending a few thousand kilometers into the interior. We argue this region cannot be described at leading order by a reduced set of equations, requiring instead the full, primitive equations for its description. It is only in this area that the mean circulation can transfer energy to the mesoscale at rates resulting in energetic equilibration. The need for full, primitive equation dynamics underscores that at least quantitatively, equilibration is poorly described by QG dynamics. 


\subsection{Energy Considerations}

The multiple-scale analysis in Appendix A breaks the circulation into the ventilated thermocline interior, the coastally confined boundary current and the open ocean separated jet, and ascribes different roles to each. Because the large-scale flows in these regions are theoretically governed by different leading-order dynamics, their energy expressions will differ. In the next section, we test this view of mean circulation energy in a numerical model. To align the upcoming numerical results with the theoretical results, we identify the time-mean, spatially averaged flow of the numerical model with the large-scale circulation described by the theory. The reasoning behind this is given in Appendix B.

We work primarily with kinetic energy statements of the time averaged mean flow. Representing the time averaging by $\overline{(\cdot)}^{t}$, fluctuations about the mean by primes $(\cdot)^{\prime}$ and vector multiplying the time averaged momentum equations by the time averaged velocities, the equation governing $\hat{K}=\left(\bar{u}^{t}\right)^{2} / 2+\left(\bar{v}^{t}\right)^{2} / 2$ becomes

$$
\frac{\partial}{\partial x_{i}}\left(\bar{u}_{i}^{t} \hat{K}\right)=-\bar{u}_{I}^{t} \frac{\partial}{\partial x_{I}} \bar{p}^{t}-\bar{\epsilon}^{t}+\frac{\partial}{\partial x_{i}} v \frac{\partial}{\partial x_{i}} \hat{K}-\bar{u}_{I}^{t} \frac{\partial}{\partial x_{i}} \overline{u_{i}^{\prime} u_{I}^{\prime}}
$$

where $\bar{p}^{t}$ is mean pressure, Einstein summation notation has been used, lower case $i$ spans all three dimensions and upper case $I$ denotes only horizontal dimensions. The quantity

$$
\bar{\epsilon}^{t}=v \frac{\partial}{\partial x_{i}} \bar{u}_{I}^{t} \frac{\partial}{\partial x_{i}} \bar{u}_{I}^{t}
$$

represents dissipation.

Manipulations involving the hydrostatic balance (see Appendix C) lead eventually to

$$
\frac{\partial}{\partial x_{i}}\left(\bar{u}_{i}^{t}\left(\hat{K}+\bar{p}^{t}\right)+{\overline{u_{i} h}}^{t}-v \frac{\partial}{\partial x_{i}} \hat{K}\right)=-\bar{\epsilon}^{t}-\bar{u}_{I}^{t} \frac{\partial}{\partial x_{i}} \overline{u_{i}^{\prime} u_{I}^{t}}-\overline{w^{\prime} b^{\prime}}{ }^{t}
$$

where $h$ is dynamic enthalpy (Young, 2010)

$$
h=\int_{P-o}^{P} \frac{b(\theta, S, P)}{g \rho_{o}} d P
$$

and ${\overline{w^{\prime} b^{\prime}}}^{t}$ denotes the vertical buoyancy flux associated with the eddies. A full derivation of 3 appears in Appendix C.

The left hand side of 3, being a divergence, becomes a statement about boundary fluxes when integrated over some volume. The right hand side, in addition to dissipation, describes processes by which the mean and eddy fields can exchange energy within the volume of integration. In the following, we will often refer to the total eddy exchange as MEC $=$ BMEC + CMEC, with

$$
\begin{aligned}
\text { BMEC } & =-\bar{u}_{I}^{t} \frac{\partial}{\partial x_{i}} \overline{u_{i}^{\prime} u_{I}^{\prime}}, \\
\mathrm{CMEC} & =-\overline{w^{\prime} b^{\prime}}, \\
\mathrm{MEC} & =-\bar{u}_{I}^{t} \frac{\partial}{\partial x_{i}} \overline{u_{i}^{\prime} u_{I}^{\prime}}{ }^{t}-\overline{w^{\prime} b^{\prime}}{ }^{t}(=\mathrm{BMEC}+\mathrm{CMEC}) .
\end{aligned}
$$

The quantity BMEC (Barotropic Mean to Eddy Conversion) is associated with eddy momentum flux divergence as a force on the mean momentum and CMEC (baroClinic Mean to Eddy Conversion) represents eddy conversions of potential energy to mean kinetic energy, often associated with baroclinic instability.

Forming a kinetic energy equation for the eddy field by subtracting the mean horizontal momentum equations from the full momentum equations, vector multiplying the result by perturbation velocity and 
averaging shows that eddy kinetic energy (defined by ${\overline{K^{\prime}}}^{t}=0.5\left(\overline{u^{\prime 2}+v^{\prime 2}}\right)$ ) receives energy inside a domain via -MEC, supporting the interpretation of eddy-to-mean flow exchanges.

\subsection{Regional Energy Balances}

The three general circulation regions suggested by our theory are the ventilated thermocline interior, the coastal jet and the separated jet. The interior is primarily geostrophic and forced by the wind. Upon a volume integration, the leading order energy statement there becomes (see Appendix B)

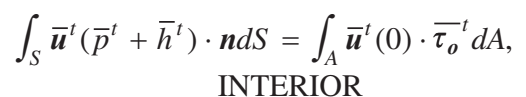

where interior viscous dissipation has been ignored, $\tau_{0}$ is the surface wind-stress, $\overline{\boldsymbol{u}}^{t}$ is the three-dimensional velocity, $\overline{\boldsymbol{u}}^{t}(0)$ is the surface velocity, and $A$ is the ocean surface part of the surface $S$ bounding the volume $V$. Equivalently, mean potential energy entering the domain $V$ is modified by the wind-stress at the surface, so that the total exiting the domain differs by the net wind work.

The coastal jet region is somewhat richer, with geostrophy for the along coast flow and the full, steady momentum balance for the cross-shore flow. The resulting energy equation, when volume integrated over the coastal region, is

$$
\int_{S} \overline{\boldsymbol{u}}^{t} \frac{\left(\bar{v}^{t}\right)^{2}}{2} \cdot \boldsymbol{n} d S=-\int_{V}\left(\bar{u}^{t} \frac{\partial}{\partial x} \bar{p}^{t}+\bar{v}^{t} \frac{\partial}{\partial y} \bar{p}^{t}\right) d V .
$$

(see Appendix B for a derivation). The interpretation of 7 is quite straight-forward: down or up pressure gradient flow will increase or decrease the kinetic energy flux.

We do not have a reduced set of equations to describe the open ocean jet, expecting instead that the full primitive equations are required, and therefore that 3 applies. Ignoring lateral viscous kinetic energy flux at the volume edge, a volume integration of 3 yields

$$
\begin{gathered}
\int_{S}\left[\overline{\boldsymbol{u}}^{t}\left(\hat{K}+\bar{p}^{t}\right)+\overline{\boldsymbol{u}}^{t}\right] \cdot \boldsymbol{n} d S=\int_{A} \overline{\boldsymbol{u}}^{t} \cdot \overline{\boldsymbol{\tau}}_{o}^{t} d S-\int_{V} \bar{\epsilon}^{t} d V+\int_{V} \operatorname{MEC} d V, \\
\text { SEPARATED JET }
\end{gathered}
$$

where we do not a priori expect any of the terms, aside from dissipation, to be small. Equation 8 argues the separated jet as a region where mean energy flux can be affected by exchange with the mesoscale because it is the only leading-order energy statement where the MEC term appears. The requirement of the full primitive equations to describe this area does suggest that the transfers will be at least quantitatively different than those described by QG dynamics, namely the instabilities will be of a generalized barotropic and baroclinic instability nature, with $O(1)$ Rossby numbers.

\subsection{Numerical Considerations}

The various contributions to 8 will be evaluated from a numerical model in the next section. Our method for diagnosing the kinetic energy budget to machine precision is outlined in Appendix D. We will refer to the various terms using the shorthand listed in Table 1, these being all the terms of the mean kinetic energy equation, as they appear in 8 .

The calculation of BMEC requires some discussion. To insure its computation to machine precision, we initialize our model configuration, described in the next section, using time averaged fields and run the model for a few time steps. The momentum equations develop a time tendency, since the mean fields are 
Table 1

Summary of the Mean Kinetic Energy Budget Terms of 8, and Their Associated Abbreviations Used in the Text

\begin{tabular}{|c|c|c|}
\hline Acronym & Formulation & Description \\
\hline DKEF & $\int_{S} \overline{\boldsymbol{u}}^{t} \hat{K} \cdot \boldsymbol{n} d S$ & Divergence of kinetic energy flux \\
\hline DPW & $\int_{S} \overline{\boldsymbol{u}}^{t} \bar{p}^{t} \cdot \boldsymbol{n} d S$ & Divergence of pressure work \\
\hline DPEF & $\int_{S} \overline{\boldsymbol{u h}}^{t} \cdot \boldsymbol{n} d S$ & Divergence of potential energy flux \\
\hline BMEC & $-\int_{V}{\overline{u_{I}}}^{t} \frac{\partial}{\partial x_{i}} \overline{u_{i}^{\prime} u_{I}^{\prime}} d V$ & $\begin{array}{l}\text { Mean-to-eddy conversion associated with eddy } \\
\text { momentum flux divergence }\end{array}$ \\
\hline CMEC & $-\int_{V} \overline{w^{\prime} b^{\prime}} d V$ & $\begin{array}{l}\text { Mean-to-eddy conversion associated with conversions } \\
\text { of potential energy to mean kinetic energy }\end{array}$ \\
\hline KEDISS & $\int_{V}(-\bar{\epsilon}+\nabla \cdot v \nabla \hat{K}) d V$ & Kinetic energy dissipation \\
\hline \multirow[t]{2}{*}{ KEDISS $=$ WW + KEDA } & \multirow[t]{2}{*}{$=\int_{-25 \mathrm{~m}}^{0 \mathrm{~m}}(-\bar{\epsilon}+\nabla \cdot v \nabla \hat{K}) d V+\int_{-1,000 \mathrm{~m}}^{-25 \mathrm{~m}}(-\bar{\epsilon}+\nabla \cdot v \nabla \hat{K}) d V$} & Wind work \\
\hline & & Kinetic energy dissipation \\
\hline
\end{tabular}

Note. The lower two lines define the respective contributions of surface wind work (WW) and interior dissipation (KEDA) to total KEDISS.

not steady solutions of the equations. Referring to 1, the kinetic energy change associated with these momentum tendencies corresponds to the quantity $\frac{t}{u_{I}} \frac{\partial}{\partial x_{i}} \overline{u_{i}^{\prime} u_{I}^{\prime}} t=-$ BMEC.

Finally, we are interested in the net mean wind forcing which, literally speaking, depends on the product of mean surface velocity and mean surface wind-stress. However, some of that energy input is locally dissipated in the surface mixed layer, and is unavailable to the general circulation. We show in Figure 1 a vertically integrated viscous energy flux profile horizontally integrated over the North Atlantic subtropical gyre region studied in this paper. The displayed quantity, $V(z)$, is defined as

$$
\begin{gathered}
V(z)=\int_{A} \int_{z}^{0} \operatorname{KEDISS} d z^{\prime} d A=\int_{A} \overline{\boldsymbol{u}(0)}{ }^{t} \cdot \bar{\tau}_{\boldsymbol{o}}^{t} d A \\
-\int_{A} \int_{z}^{0} \bar{\epsilon}^{t} d z^{\prime} d A-\int_{A} \overline{\boldsymbol{u ( z )}}{ }^{t} \cdot \overline{\tau(z)} d A,
\end{gathered}
$$

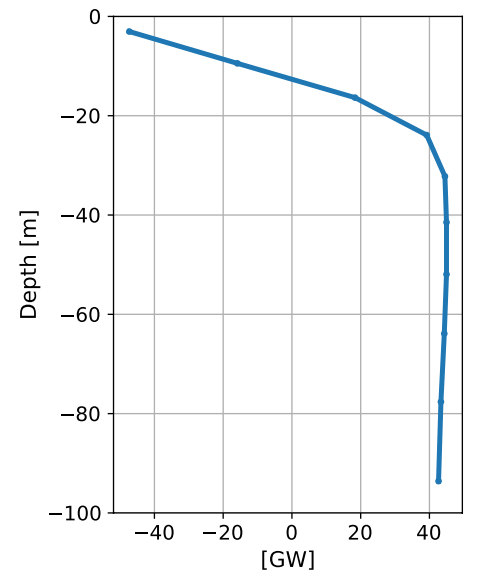

Figure 1. A net viscous energy flux profile integrated over the region of the subtropical North Atlantic gyre. The rapid increase moving downward away from the surface indicates the region of extreme mixed layer dissipation. At about $25 \mathrm{~m}$, the profile settles to a slowly varying value of roughly $41 \mathrm{GW}$, which we take as the network from the wind available to the wind-driven circulation. where lateral viscous fluxes have been ignored and $\tau=v \boldsymbol{u}_{z}$. The topmost value (at the base of the first grid cell) in Figure 1 is the total wind work adjusted for dissipation and flux through the bottom of the grid cell. Moving downward, a rapid increase in $V(z)$ is seen, which is associated with decreasing local mixed layer dissipation and flux through the deeper grid cell face. The profile at depths greater than roughly $25 \mathrm{~m}$ settles to a slowly evolving structure, consistent with the profile being out of the intense surface dissipation zone. We take the energy flux at a depth of roughly $25 \mathrm{~m}$, computed according to 9 , as indicative of the net wind input to the large-scale circulation, and refer to it in the discussion below as "wind work" (WW). For the profile in Figure 1, this is a value of roughly $41 \mathrm{GW}$. Further, we will write KEDISS = WW + KEDA

$$
\begin{gathered}
\mathrm{WW}=V(-25 \mathrm{~m})=\int_{A} \int_{-25 \mathrm{~m}}^{0} \mathrm{KEDISS} d z^{\prime} d A \\
\mathrm{KEDA}=\int_{A} \int_{-z_{o}}^{-25 \mathrm{~m}} \mathrm{KEDISS} d z^{\prime} d A,
\end{gathered}
$$

where $-z_{0}$ is $1,000 \mathrm{~m}$ for reasons to be discussed shortly. 


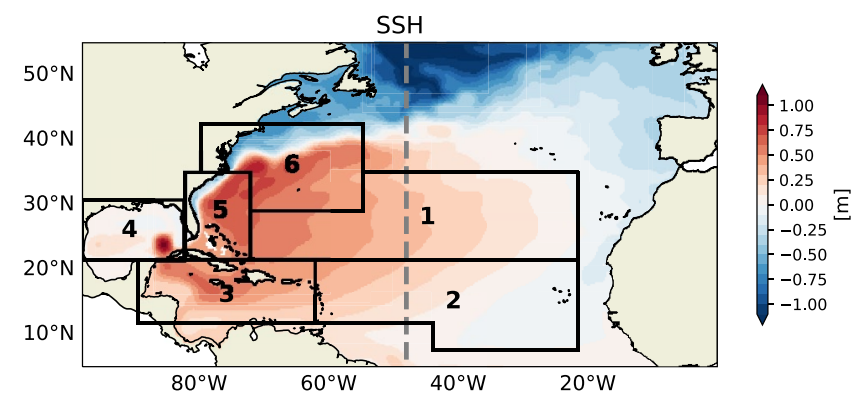

Figure 2. 1970-1995 time mean sea surface height in the North Atlantic sector of our model. The Gulf Stream and its open ocean extension are clearly visible as are other well-known Atlantic features. The meridional dashed gray line denotes the location of a north-south potential density transect appearing in the next figure. Six boxes appear which enclose the regions over which the energy equation is averaged.

\section{Numerical Results}

\subsection{Model Description}

We analyze a regional configuration of the Massachusetts Institute of Technology General Circulation Model (MITgcm, Marshall et al., 1997). The horizontal resolution is $1 / 12^{\circ}$ and 46 layers are used in the vertical, with vertical spacing ranging from $6 \mathrm{~m}$ at the surface to $250 \mathrm{~m}$ at depth. A resolution of $1 / 12^{\circ}$ is generally recognized as "eddy resolving," although the eddy activity is undoubtedly underestimated (Chassignet \& Xu, 2017). Boundary conditions at $55^{\circ} \mathrm{N}$ and $20^{\circ} \mathrm{S}$ and at the Strait of Gibraltar are extracted from the $1 / 12^{\circ}$ global Drakkar run ORAC12.L46-MJM88 (Molines et al., 2014; Serazin et al., 2015). Topography comes from the ORCA12. L46 configuration, which is a combination of ETOPO1 for the deep ocean and GEBCO_08 for shallow areas (Molines et al., 2014), a choice which also provided consistency with the boundary conditions. The model uses the modified UNESCO equation of state (Jackett \& McDougall, 1995).

Tracer and momentum equations employed both Laplacian and biharmonic operators with coefficients of $20 \mathrm{~m}^{2} \mathrm{~s}^{-1}$ and $-10^{10} \mathrm{~m}^{4} \mathrm{~s}^{-1}$, respectively. At the surface, the ocean model was coupled to the atmospheric boundary layer package, CheapAML (Deremble et al., 2013), which computes wind-stress, latent flux, and sensible flux according to the COARE3 (Fairall et al., 2003) flux formula, as well as upwelled long-wave radiation. The Drakkar forcing data set, version 4.4 (DFS4.4) was used to produce atmospheric and radiative conditions, and precipitation from DFS5.3 was used due to higher time resolution. All atmospheric variables are strongly restored toward the prescribed DFS4.4 values over land. Surface forcing was applied every $6 \mathrm{~h}$ and open boundary conditions every 5 days.

Initial conditions were obtained after a 6 year spin-up, consisting of a 5-year long run (1958-1962) under realistic forcing, with initial conditions derived from the oceanic state of the ORCA12.L46-MJM88 configuration on January 1, 1958, followed by an additional 1-year long simulation under 1963 forcing with perturbed initial conditions. This additional year of simulation was used to produce an ensemble (Jamet et al., 2019), one realization of which is used in this work. From the subsequent January 1st state, the configuration was integrated for 50 years (1963-2012), and this study focuses on a 26-year long segment (1970-1995) of this simulation.

Figure 2 shows the sea surface height for the North Atlantic sector. The Gulf Stream appears along the U.S. eastern coast and its open ocean extension reaches out to a longitude of roughly $50^{\circ} \mathrm{W}$. There is an $\mathrm{SSH}$ imprint of the Loop Current in the Gulf of Mexico.

We have segmented the ocean into several sectors, shown in Figure 2, in which we perform our energy analysis. The six domains are referred to as the "North Interior" (box 1), the "South Interior" (box 2), the "Caribbean" (box 3), the Gulf of Mexico (box 4), the "coastal Gulf Stream" (box 5) and the "Separated Jet" (box 6). Boxes (1)-(3) capture the main body of the interior wind-driven circulation, dominated by a slow southwestward drift. The Gulf of Mexico (box 4) is in some way a transition zone between the interior and the development of the Gulf Stream jet. The Gulf Stream transit from the tip of South Florida to Cape Hatteras out to a reasonable distance from the coast is in the fifth box. The last box encompasses the region of the identifiable open ocean Gulf Stream, out roughly to a longitude of $55^{\circ} \mathrm{W}$, as indicated by surface speed.

Note that boxes 3, 4, and 5 are simple rectangles. Boxes 1, 2, and 6 are more complicated for the following reasons. We have included the so-called Gulf Stream recirculation or Worthington Gyre (Worthington, 1976) in the separated jet box as it is a feature whose existence depends on the separated jet variability and is clearly not part of the Sverdrup interior, as it the remainder of box 1. Similarly, the region housing the North Brazil Current retroflection is cut out of box 2 as those dynamics are not described well by geostrophy.

We show in Figure 3 a north-south transect of potential density taken along the meridional dashed gray line in Figure 2. We observe the signature of a well-organized baroclinic gyre with an imprint of the eastward flowing Gulf Stream extension at about $40^{\circ} \mathrm{N}$, and the bowl associated with the anticyclonic gyre south of 


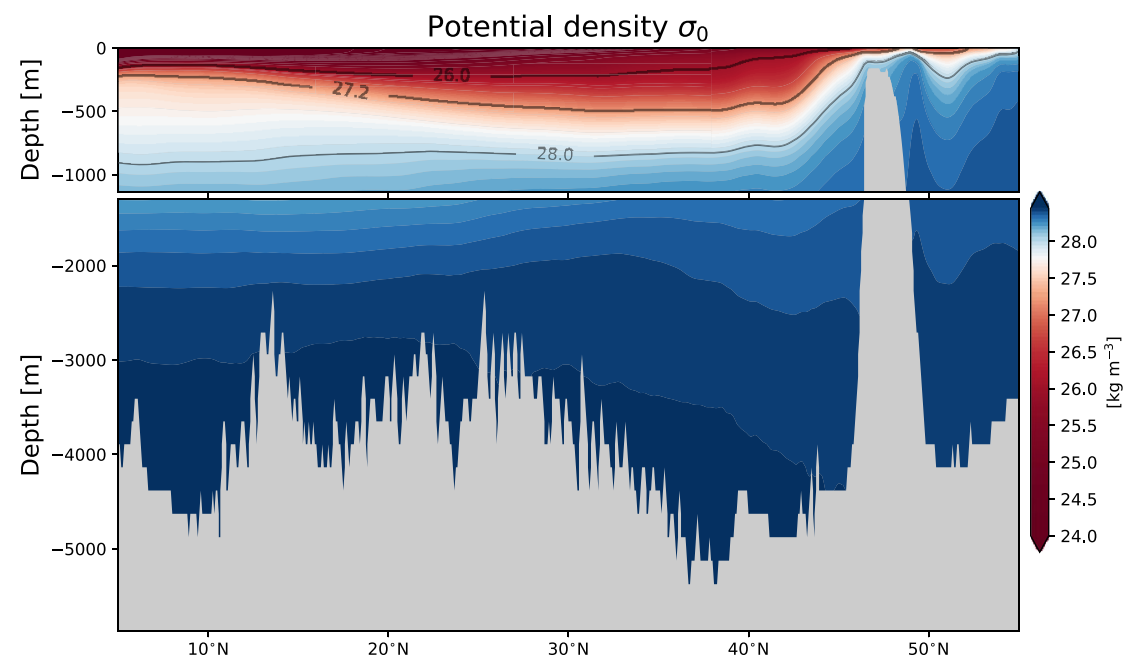

Figure 3. A transect of time mean potential density $\sigma_{0}=\left(\rho\left(\theta, S, P=P_{0}\right)-1,000\right) \mathrm{kg} \mathrm{m}^{-3}$ for $1970-1995$ through the North Atlantic at about $45^{\circ} \mathrm{W}$ (the dashed gray line in Figure 2). The Gulf Stream density structure appears at $40^{\circ} \mathrm{N}$. The wind-driven circulation penetrates to depths of roughly $1 \mathrm{~km}$.

the Gulf Stream. The nature of the isopycnals changes at a depth of roughly $1 \mathrm{~km}$, which we will take to be the vertical extent the wind-driven circulation. Consequently, the energy integrals discussed later extend to $1,000 \mathrm{~m}$ in order to capture the wind-driven energetics.

The mean wind work distribution for the area appears in Figure 4. The values are generally positive, indicating a net driving of the local circulation by the wind. We note that the open ocean wind work inside of box 1 is relatively small. This is due to the transition of the mean wind from being westerly to easterly in this latitudinal band.

In an integral sense we are motivated by a desire to understand how and where energy input to the general circulation from the wind exits the general circulation to the mesoscale. The form of 8 is such that MEC represents the mesoscale sink, and we expect the net wind energy input over the gyre to be balanced by the net value of MEC integrated over the gyre. In the previous section, we argued the net energy input over the subtropical North Atlantic (all boxes in Figure 4) is 41 GW. We here note that the MEC integrated over the same domain and to a depth of $1,000 \mathrm{~m}$ gives a value of roughly $43 \mathrm{GW}$ which, while not identical to the total wind forcing, suggests this quantity brings the general circulation approximately into energetic equilibrium.

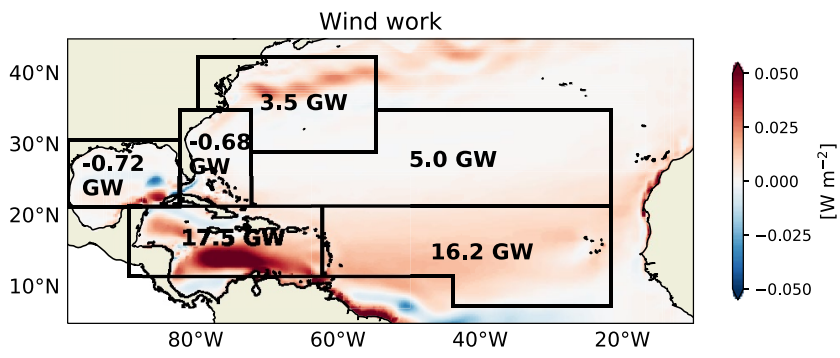

Figure 4. The distribution for surface Wind Work (WW, $\boldsymbol{u} \cdot \tau)$ over the North Atlantic. The values are generally positive. The numbers are the net wind work in each of the boxes appearing in Figure 2. We note for later discussion that the central part of the gyre in box 1 is a region of minimal wind work, due to the reversal of the winds from primarily westerly to easterly.

\subsection{Regional Kinetic Energy Considerations}

Our strategy has been to consider the integrals in 8 over the boxes in Figure 2 and down to depths of $1 \mathrm{~km}$ to confine the energy budgets to the wind-driven circulation. All of the quantities in 8 have been evaluated, from which we infer leading-order balances. A summary of the results appears in Table 2. For simplicity, DPW and DPEF have been combined together. They appear separately in Table 2 from which it is seen that always have opposite signs and comparable magnitudes; we interpret their sum as the "effective" potential energy flux. Similarly, BMEC and CMEC are combined together since they both contribute to mean-to-eddy energy exchanges.

The evaluation of 8 in the interior boxes 1 through 4 breaks down respectively as 
Table 2

Regional Kinetic Energy Contributions (GW), Evaluated as the Volume Integration of the Various Terms of the Mean Kinetic Energy Budget Listed in 1

\begin{tabular}{|c|c|c|c|c|c|c|}
\hline Box name & $\mathrm{N}$ Int & S int & Caribbean & GOM & Coastal & Jet \\
\hline Box number & 1 & 2 & 3 & 4 & 5 & 6 \\
\hline \multicolumn{7}{|l|}{ Formulation } \\
\hline $\mathrm{DKEF}=\int_{S} \overline{\boldsymbol{u}}^{t} \hat{K} \cdot \boldsymbol{n} d S$ & 0.0 & 0.03 & 4.4 & 0.3 & 19.8 & -26.4 \\
\hline $\mathrm{DPW}=\int_{S} \overline{\boldsymbol{u}}^{t} \bar{p}^{t} \cdot \boldsymbol{n} d S$ & 131.0 & 233.0 & 199 & -75.6 & -186.0 & 92.4 \\
\hline $\mathrm{DPEF}=\int_{S} \overline{\boldsymbol{u}}^{t} \cdot \boldsymbol{n} d S$ & -131.6 & -214.0 & -191 & 67.5 & 145.0 & -84.5 \\
\hline $\mathrm{DPW}+\mathrm{DPEF}$ & -0.6 & 19.0 & 8 & -8.1 & -41.0 & 7.9 \\
\hline $\mathrm{WW}=\int_{A} \overline{\boldsymbol{u}}^{t} \mathrm{I}_{z=0} \cdot \overline{\boldsymbol{\tau}}_{o}^{t} d S$ & 5.0 & 16.2 & 17.5 & -0.72 & -0.68 & 3.5 \\
\hline $\mathrm{KEDA}=-\int_{V} \bar{\epsilon}^{t} d V$ & -0.01 & -0.06 & -2.20 & -3.0 & -6.80 & -0.76 \\
\hline $\mathrm{BMEC}=-\int_{V} \bar{u}_{I}^{t} \frac{\partial}{\partial x_{i}} \overline{u_{i}^{\prime} u_{I}^{\prime}} d V$ & -0.2 & 5.3 & 1.7 & -4.4 & -11.9 & -14.8 \\
\hline $\mathrm{CMEC}=-\int_{V} \overline{w^{\prime} b^{\prime}} d V$ & -5.4 & -2.1 & -4.2 & 0.27 & -1.4 & -6.5 \\
\hline MEC & -5.6 & 3.2 & -2.5 & -4.1 & -13.3 & -21.3 \\
\hline
\end{tabular}

Note. The fourth line reflects that DPW and DPEF are always of opposite sign and of comparable magnitude, such that we interpret their sum as the "effective" potential energy flux. Similarly, BMEC and CMEC are combined together in the last line of the table. These numbers also appear in 11-13.

$$
\begin{aligned}
\int_{S}\left(\overline{\boldsymbol{u}}^{t} \hat{K}+\overline{\boldsymbol{u}}^{t} \bar{p}^{t}+\overline{\boldsymbol{u} h}^{t}\right) \cdot \boldsymbol{n} d S & =\int_{S} \overline{\boldsymbol{u}}_{o}^{t} \cdot \overline{\boldsymbol{\tau}}_{o}^{t} d A-\int_{V} \bar{\epsilon}^{t} d V+\int_{V} \mathrm{MEC} d V \\
\mathrm{DKEF}+\mathrm{DPW}+\mathrm{DPEF} & =\mathrm{WW}+\mathrm{KEDA}+\mathrm{MEC} \\
0.0 \mathrm{GW}-0.6 \mathrm{GW} & =5.0 \mathrm{GW}-0.01 \mathrm{GW}-5.6 \mathrm{GW} \\
0.03 \mathrm{GW}+19.0 \mathrm{GW} & =16.2 \mathrm{GW}-0.06 \mathrm{GW}+3.2 \mathrm{GW} \\
4.4 \mathrm{GW}+8.0 \mathrm{GW} & =17.5 \mathrm{GW}-2.2 \mathrm{GW}-2.5 \mathrm{GW} \\
0.3 \mathrm{GW}-8.1 \mathrm{GW} & =-0.72 \mathrm{GW}-3.0 \mathrm{GW}-4.1 \mathrm{GW}
\end{aligned}
$$

Quite noticeable is that DKEF is very small in boxes 1, 2, and 4. It is somewhat larger in box 3 but, as shown later, all the divergence comes up against the Caribbean island arc near $60^{\circ} \mathrm{W}$, where the circulation compacts as it flows through the island topographic obstacles. In general, away from topography, kinetic energy is an unimportant player in the interior, which is expected from a largely geostrophic flow. In box 2 , the net wind work is largely balanced by the effective potential energy flux divergence. Away from the archipelago, the same holds for the Caribbean box. A seeming exception is box 1, where potential energy divergence is very weak, and wind work is largely balanced by MEC. On the other hand, this is the weakest area of wind forcing of the subtropical gyre, with an input of $5 \mathrm{GW}$, as compared to $17.5 \mathrm{GW}$ in box 3 . The relative change in boxes 2 and 3 in wind work is matched by the changes in net potential energy flux divergence, whereas MEC remains comparable.

The weak values of MEC for boxes 1-3 are consistent with the predictions of ventilated thermocline theory. There are eddies and mesoscale events in the ocean interior, but the mean to eddy energy conversions associated with them are of secondary importance. Generally, net potential energy flux divergence balances wind work. MEC is also weak in the Gulf of Mexico although it is not a region of ventilated thermocline dynamics (wind work is negligible).

Moving to box 5, the coastal Gulf Stream, the energy contributions are 

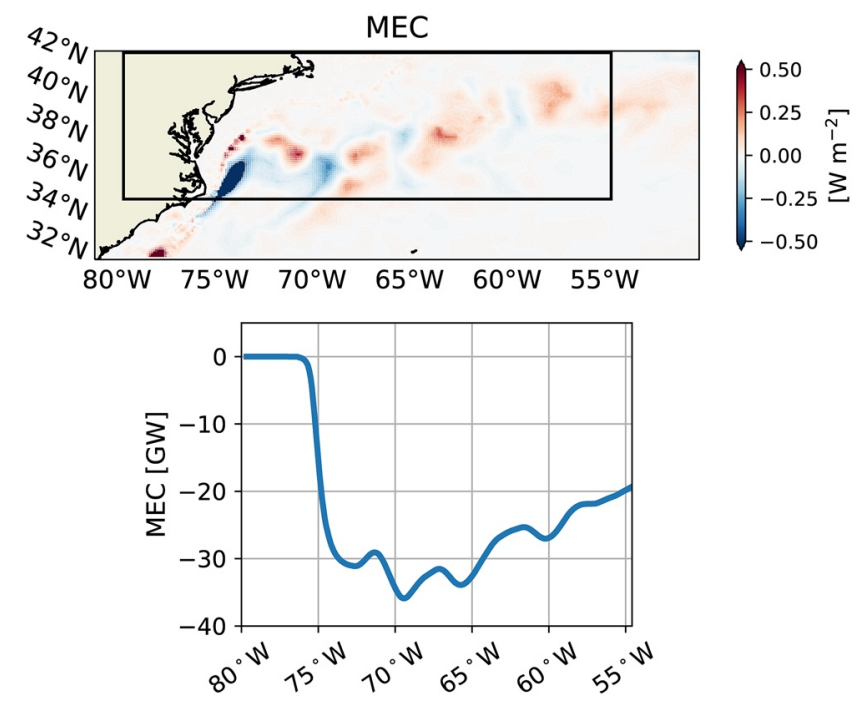

Figure 5. A map of MEC integrated to $1,000 \mathrm{~m}$ in the vicinity of the jet separation point appears in the top panel. The outlines of the U.S. east coast appear in black. The contours are in $\mathrm{W} \mathrm{m}^{-2}$. A large maximum in loss to the mesoscale is seen immediately downstream of Cape Hatteras and followed by a sequence of undulations that decay with distance downstream. The bottom panel contains a plot of the cumulative net conversion from mean to eddies starting at the western end of the box on top panel. Note the rapid decline to values in excess of $-35 \mathrm{GW}$ followed by a slower increase.

$$
\begin{array}{rlrl}
\int_{S}\left(\overline{\boldsymbol{u}}^{t} \hat{K}+\overline{\boldsymbol{u}}^{t} \bar{p}^{t}+\overline{\boldsymbol{u}}^{t}\right) \cdot \boldsymbol{n} d S & =\int_{S} \overline{\boldsymbol{u}}_{o}^{t} \cdot \overline{\boldsymbol{\tau}}_{o}^{t} d A-\int_{V} \bar{\epsilon}^{t} d V+\int_{V} \mathrm{MEC} d V \\
\mathrm{DKEF}+\mathrm{DPW}+\mathrm{DPEF} & =\mathrm{WW}+\mathrm{KEDA}+\mathrm{MEC} \\
19.8 \mathrm{GW}-41 \mathrm{GW} & = & -0.68 \mathrm{GW}-6.8 \mathrm{GW}-13.3 \mathrm{GW}
\end{array}
$$

It is clear the regional energy structure differs considerably from the open ocean. First, the net wind work is small, consistent with western boundary scaling. The most important contrast, however, is that the net potential energy flux divergence $(-41 \mathrm{GW})$ has changed sign relative to the interior, now being negative. Further, this value of $-41 \mathrm{GW}$ is quite close in value to $\bar{u}^{t} \bar{p}_{x}^{t}+\bar{v}^{t} \bar{p}_{y}^{t}$, which is related to kinetic energy growth by flow down the mean pressure gradient B18. Another major change is that the kinetic energy flux divergence has now become of leading-order importance. Further, it is positive, indicative of an increase in total flux as one proceeds from the tip of South Florida to Cape Hatteras. While its growth does not account completely for the net potential energy flux, it is comparable (roughly 50\%). These two points are supportive of the multiple scales boundary layer analysis discussed in the previous section. We do note, however, that dissipation and mean to eddy energy conversion are both nonnegligible in the region. The importance of dissipation is consistent with the fact that topography everywhere interacts with the current in this region. The mean to eddy energy conversion is smaller than the growth in kinetic energy flux by roughly a factor of 2, although it is still a sizable contribution to the overall energy budget. This does not appear in the theoretical analysis. However, we note that the principle locations of MEC in box 5 are at topographic irregularities, the largest being the Charleston Bump, and the theory neglected along stream topographic variations.

In the separated jet zone, box 6 , the contributions to the energy equation parse according to

$$
\begin{aligned}
\int_{S}\left(\overline{\boldsymbol{u}}^{t} \hat{K}+\overline{\boldsymbol{u}}^{t} \bar{p}^{t}+\overline{\boldsymbol{u}}^{t}\right) \cdot \boldsymbol{n} d S & =\int_{S} \overline{\boldsymbol{u}}_{o}^{t} \cdot \overline{\boldsymbol{\tau}}_{o}^{t} d A-\int_{V} \bar{\epsilon}^{t} d V+\int_{V} \mathrm{MEC} d V \\
\mathrm{DKEF}+\mathrm{DPW}+\mathrm{DPEF} & =\mathrm{WW}+\mathrm{KEDA}+\mathrm{MEC} \\
-26.4 \mathrm{GW}+7.9 \mathrm{GW} & =3.5 \mathrm{GW}-0.76 \mathrm{GW}-21.3 \mathrm{GW}
\end{aligned}
$$

The net potential energy flux $(7.9 \mathrm{GW})$ has changed sign from the coastal Gulf Stream jet and is consistent with a flow up the mean pressure gradient. The kinetic energy flux divergence is now negative, indicating a local decrease in kinetic energy flux, and both these quantities are leading-order contributors to the regional balance. This is consistent with a recycling of energy, namely energy released to kinetic energy by an effectively down-gradient flow along the coast is now moving back up-gradient into potential energy. Equally significant, however, is MEC, which is consistent with a loss of mean energy to the eddy field. We show in Figure 5 (top) a map of net MEC integrated over the upper 1,000 $\mathrm{m}$ in the region of the Gulf Stream separation. The structure here is dominated by a sequence of alternating highs and lows that decrease in amplitude with increasing separation from Cape Hatteras. The cumulative net conversion moving from the west side of the box to the east side appears in Figure 5 (bottom), where it is seen that immediately downstream of Cape Hatteras loss to the mesoscale grows dramatically to losses greater than - 35 GW. Thereafter, there is a slow increase in amplitude, consistent with a conversion back to the mean field from the mesoscale and a decreasing tendency for the Gulf Stream to meander. The net effect of -14 GW reflects a considerable energy exchange in both directions although the final value represents a loss of mean energy to the mesoscale field.

A final comment is that the total mean to eddy conversion occurring over the coastal and separated Gulf Stream is roughly $-34.6 \mathrm{GW}$ (BMEC + CMEC, boxes 5, 6) which accounts for most of the total wind 
forcing of the subtropical North Atlantic region, $\approx 41 \mathrm{GW}$ (WW, all boxes). Given that the total MEC integrated over the broader North Atlantic is roughly -43 GW (BMEC + CMEC, all boxes), the coastal and separated jets clearly dominate the conversion. We do not claim that these numbers match each other, merely that their rough correspondence argues that bulk of the energetics equilibration of the North Atlantic mean circulation occurs in these zones, with net mean forcing leaving the mean flow to enter the mesoscale field.

\subsection{Potential to Kinetic Energy Conversions}

It is of interest that the two conversion terms, BMEC and CMEC, are of comparable strength in the subtropical North Atlantic. In the separated jet box 6, BMEC is $-14.8 \mathrm{GW}$, while CMEC is $-6.5 \mathrm{GW}$. What is notable about this is CMEC is usually associated with baroclinic instability, and is thought to be the dominant geostrophic instability in the ocean. While it is more widespread in distribution over the gyre than BMEC, certainly in the separated jet zone, it is weaker than the barotropic processes. Over the entire North Atlantic gyre, $\mathrm{BMEC}=-24.3 \mathrm{GW}$ and $\mathrm{CMEC}=-19.3 \mathrm{GW}$ (all boxes).

\subsection{Is the General Circulation Generally Inertial?}

We now point out one last feature of the general circulation kinetic energy budget that falls slightly out of the above framework, but which we feel merits mention. From 3, the net flux of mean potential energy in the interior is given by

$$
\text { mean PE flux }=\overline{\boldsymbol{u}}^{t} \bar{p}^{t}+\overline{\boldsymbol{u} h}^{t}
$$

to the neglect of diabatic and nonhydrostatic quantities. The analysis of the open ocean results have argued that the divergence of the effective potential energy flux, that is, DPW + DPEF, is roughly in balance with the open ocean wind energy input, in keeping with ventilated thermocline dynamics. What we want to emphasize is that this divergence is small compared to the amplitude of the participant fluxes. This appears in Figure 6 which plots the latitudinal dependence of the net meridional flux $\bar{v}^{t} \bar{p}^{t}+\bar{v}^{t}$ integrated over longitude and the longitudinal dependence of zonal flux $\bar{u}^{t} \bar{p}^{t}+\overline{u h}^{t}$ integrated over latitude in boxes 1 and 2. The total fluxes are as large as $-70 \mathrm{GW}$, that is, much larger in value compared to either the local wind energy inputs or net divergences $(O(10 \mathrm{GW}))$. The implication of this is that the interior geostrophic ventilated thermocline is dominantly recirculating energy. This is the earmark of an inertial, interior, geostrophic circulation.

\section{Summary}

We have attempted in this paper to construct a comprehensive energy picture of the mean subtropical gyres. This is motivated by the current interest in detailing ocean energy broadly speaking and the idea that approximately $\leq 1$ TW flows from the large-scale, low-frequency winds to the oceanic mesoscale. We argue by means of a multiple-scale analysis that the interior receives the bulk of the wind forcing, but is not the location where the mean circulation loses energy. We then analyze an anisotropic western boundary current that leads to coastal jets fed by the ventilated thermocline. These inertial jets accelerate at the expense of pressure work. Coastal jets are connected downstream to open ocean jets that require full primitive equation dynamics for their description. As such they are the only places that can involve mean flow and mesoscale interactions.

We next analyze an eddy-resolving model of the North Atlantic, identifying the time mean flow with the large-scale flow of the theory. We find the ocean interior is consistent with the ventilated thermocline and exhibits minimal energy loss to the mesoscale while largely recycling energy. In the coastal Gulf Stream, kinetic energy flux grows due to net pressure work, which is in agreement with our theory. Our analysis also finds nontrivial dissipation and mean to eddy energy conversion, but these are smaller than the exchanges between kinetic energy and pressure work. The coastal mesoscale generation appears to have topographic origins. The open ocean Gulf Stream emerges as the location of the largest and most dynamic 

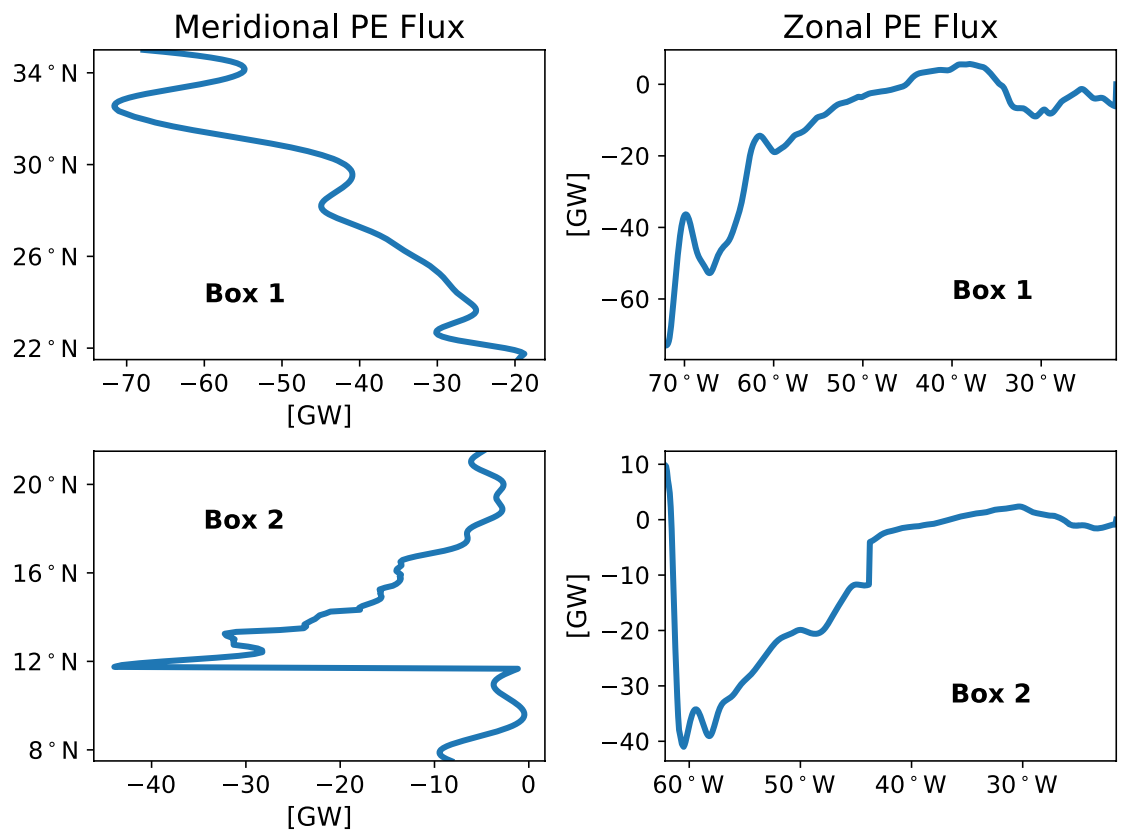

Figure 6. Zonal and meridional effective potential energy fluxes integrated across interior boxes 1 and 2 . The total fluxes are several tens of GW which is significantly larger that either the local wind energy inputs or the effective potential energy flux divergences. Note also that the zonal flux in box 2 drops off dramatically at the Caribbean archipelago near $60^{\circ} \mathrm{W}$. This feeds the increase in DKEF seen in box 2.

mean-to-eddy energy conversion, involving exchanges with eddies of both signs and an associated flow up the mean pressure gradient. The dynamics of this area are rich and at best only qualitatively described by QG dynamics.

Fofonoff (1981) in a review of the Gulf Stream system proposed a North Atlantic energy structure much like that seen here. He suggested that wind forcing in the interior largely acted to increase the large-scale potential energy flux, and that Gulf Stream acceleration came at the expense of the large-scale pressure gradient. He also identified the separated jet as a region of energy recycling caused by mean flow in the direction of increasing pressure. To this picture, we add an eddying component to the open ocean Gulf Stream and suggest the energy recycling includes the ocean interior as well. That Fofonoff was able to infer this structure from the observations and model results available over 30 years ago is testament to his remarkable insight into ocean dynamics.

We argue in this paper that the separated jet is the principal area of mesoscale generation by geostrophic instabilities of the mean flow. Inasmuch as wind-driven gyres are a common feature of the mid-latitude general circulation, this analysis may well apply beyond the North Atlantic where the numerical study has been confined. To further clarify oceanic wind-driven energy, focused studies in such regions might well be fruitful avenues for pursuit.

\section{Appendix A: Multiple-Scale Analysis of the Wind-Driven Circulation}

The purpose of this appendix is to present the details of the multiple-scale analysis resulting in our segmented view of the general circulation. The interior analysis appears first, followed by the coastal jet analysis. We begin with the hydrostatic equations of motion written in buoyancy coordinates (Bleck \& Chassignet, 1994), assuming a linear equation of state in which we have only a single thermodynamic variable. 


$$
\begin{gathered}
\frac{\partial}{\partial t} u+u \frac{\partial}{\partial x} u+v \frac{\partial}{\partial y} u-f v=-\frac{\partial}{\partial x} M+\frac{1}{\frac{\partial}{\partial b} z} \nabla \cdot D^{x} \\
\frac{\partial}{\partial t} v+u \frac{\partial}{\partial x} v+v \frac{\partial}{\partial y} v+f u=-\frac{\partial}{\partial y} M+\frac{1}{\frac{\partial}{\partial b} z} \nabla \cdot D^{y} \\
\frac{\partial}{\partial b} M=-z \\
\frac{\partial}{\partial t} \frac{\partial}{\partial b} z+\frac{\partial}{\partial x}\left(u \frac{\partial}{\partial b} z\right)+\frac{\partial}{\partial y}\left(v \frac{\partial}{\partial b} z\right)=0,
\end{gathered}
$$

where $M$ is the Montgomery potential, $M=p-b z$ where $p$ is pressure (Montgomery, 1937), $b$ is buoyancy, $\boldsymbol{D}^{x}, \boldsymbol{D}^{y}$ are zonal and meridional, three dimensional, viscous fluxes, diffusive effects are ignored and the notation is otherwise standard. The equations are then nondimensionalized using the planetary length scale, $L_{\beta}=f_{o} / \beta$ (where $f_{o}$ and $\beta$ are typical values for the Coriolis parameter and its meridional derivative) and a specified surface buoyancy range. The buoyancy range is used to define the deformation radius, from which a time scale representing the time needed for a planetary wave to cross the basin is derived (see A4). Pressure scaling is obtained from the hydrostatic balance using the Welander (1959) thermocline thickness scaling. After nondimensionalization, A1 become

$$
\begin{gathered}
R_{o}\left(\frac{\partial}{\partial T} u+u \frac{\partial}{\partial X} u+v \frac{\partial}{\partial Y} u\right)-f v=-\frac{\partial}{\partial X} M \\
R_{o}\left(\frac{\partial}{\partial T} v+u \frac{\partial}{\partial X} v+v \frac{\partial}{\partial Y} v\right)+f u=-\frac{\partial}{\partial Y} M \\
\frac{\partial}{\partial b} M=-z \\
\frac{\partial}{\partial T} \frac{\partial}{\partial b} z+\frac{\partial}{\partial X}\left(u \frac{\partial}{\partial b} z\right)+\frac{\partial}{\partial Y}\left(v \frac{\partial}{\partial b} z\right)=0,
\end{gathered}
$$

where $R_{o}=U /\left(f_{o} L_{\beta}\right)$ is the Rossby number (with velocity scale $U$ ) and frictional effects have been ignored. The quantity $f$ is now nondimensionalized by $f_{o}$ but a function of meridional location $Y$. The independent variables $X, Y$ denote zonal and meridional location as scaled by the planetary length scale and $T$ nondimensional time as scaled on the wave basin transit time. Defining the deformation radius, $R_{d}$, as

$$
R_{d}^{2}=\frac{g \Delta \rho H}{\rho_{o} f_{o}^{2}}=\frac{\Delta b H}{f_{o}^{2}}
$$

where $\Delta \rho$ is the assumed known surface density variation and $\rho_{0}$ a reference density, the Rossby number turns out to be

$$
R_{o}=\frac{R_{d}^{2}}{L_{\beta}^{2}} .
$$

At leading order, A3 return the PG equations. To enrich them in a search for their interactions with smaller deformation radius motions, a standard multiple scales approach is used, which involves replacing all the derivatives with their multiple scale versions, that is,

$$
\frac{\partial}{\partial X} \rightarrow \frac{1}{\delta} \frac{\partial}{\partial x}+\frac{\partial}{\partial X}
$$

where $\delta$ is the ratio of the deformation radius to the planetary scale

$$
\delta=\frac{R_{d}}{L_{\beta}}=\sqrt{R_{o}},
$$


and the lower case $x$ now denotes a nondimensional small spatial scale. Substitutions like A5 are carried out into A3 in both space and time, reflecting that deformation scale dynamics are faster and shorter in scale than planetary motions. A key point here is that the planetary scale and the deformation scale have been introduced isotropically into the equations, that is, mesoscale variability is assumed to scale with the deformation radius in both horizontal directions, and PG dynamics with $L_{\beta}$ in a similar way.

The next step is to expand all variables in the small parameter $\delta$ and exploit the implied scale separations in space and time by averaging procedures. It turns out at leading order, the Montgomery potential depends only on the buoyancy, the long spatial scale and the slow temporal scale, $M_{o}=M_{o}(X, Y, b, T)$, while geostrophy involves both large and small scales

$$
\begin{aligned}
f(Y) u_{o} & =-\frac{\partial}{\partial y} M_{1}-\frac{\partial}{\partial Y} M_{o} \\
-f(Y) v_{o} & =-\frac{\partial}{\partial x} M_{1}-\frac{\partial}{\partial X} M_{o}
\end{aligned}
$$

where $M_{1}$ is the next order correction to the Montgomery potential. Consistent with A7, deformation radius buoyancy anomalies are seen to be order Rossby number relative to the mean state stratification. A similar approach to the continuity equation, after expansion in $\delta$, leads to

$$
\begin{gathered}
\frac{\partial}{\partial T} \frac{\partial}{\partial b} z_{o}+\frac{\partial}{\partial X}\left(u_{o} \frac{\partial}{\partial b} z_{o}\right)+\frac{\partial}{\partial Y}\left(v_{o} \frac{\partial}{\partial b} z_{o}\right)= \\
-\frac{\partial}{\partial t} \frac{\partial}{\partial b} z_{1}-\frac{\partial}{\partial x}\left(u_{1} \frac{\partial}{\partial b} z_{o}+u_{o} \frac{\partial}{\partial b} z_{1}\right)-\frac{\partial}{\partial y}\left(v_{1} \frac{\partial}{\partial b} z_{o}+v_{o} \frac{\partial}{\partial b} z_{1}\right) .
\end{gathered}
$$

It is here that the lack of QG feeback onto the PG structure becomes apparent. This is illustrated by averaging A8 over spatial scales large compared to the deformation radius, but small compared to the planetary scale, that is,

$$
\bar{\zeta}=\frac{1}{A} \int_{A} \zeta d x d y
$$

where the overbar denotes a spatial average and $A$ represents an area whose length scales in the meridional and zonal directions, $L_{A}$, are much larger than the deformation radius used to nondimensionalize the short spatial scales. As explained in P84, such an integration over a divergence operator depending on the short spatial scales leads to a result that is $O\left(R_{d} / L_{A}\right) \ll 1$ asymptotically small, while quantities depending on the large-scale variables $X, Y$ are untouched. Mathematical consistency requires such quantities must be separately equated to zero. This averaging is a standard multiple-scales procedure and should not be confused with other averaging procedures often employed in buoyancy coordinates, like "thickness weighted averaging” (TWA), or "transformed Eulerian mean" averaging (Gent \& McWilliams, 1990; Plumb \& Ferrari, 2005; Young, 2012), the objectives of which are different than those here.

The result of the averaging A10 is

$$
\frac{\partial}{\partial T} \frac{\partial}{\partial b} z_{o}+\frac{\partial}{\partial X}\left(\overline{u_{o}} \frac{\partial}{\partial b} z_{o}\right)+\frac{\partial}{\partial Y}\left(\overline{v_{o}} \frac{\partial}{\partial b} z_{o}\right)=-\overline{\frac{\partial}{\partial t} \frac{\partial}{\partial b} z_{1}} .
$$

The left-hand side of A11 is independent of the fast time, $t$, and so to suppress secular growth in time both sides must vanish independently. In addition, spatially averaging A7 over lengths large compared to the deformation radius shows the averaged velocities are determined entirely by the leading-order Montgomery potential. Hence PG and the ventilated thermocline are obtained, but no connection to the QG fields is found.

In contrast, pushing the analysis further to clarify the QG dynamics returns 


$$
\frac{\partial}{\partial t} q+\overline{u_{o}} \frac{\partial}{\partial x} q+\overline{v_{o}} \frac{\partial}{\partial y} q+\frac{1}{f} J\left(M_{1}, q\right)+\frac{\frac{\partial}{\partial b} z_{o}}{f} \hat{J}\left(M_{1}, \frac{f}{\frac{\partial}{\partial b} z_{o}}\right)=0
$$

where

$$
q=\frac{1}{f} \nabla^{2} M_{1}+f \frac{\frac{\partial^{2}}{\partial b^{2}} M_{1}}{\frac{\partial}{\partial b} z_{o}}
$$

is recognized as the usual QG potential vorticity, $J$ denotes a Jacobian based on the fast spatial scales and $\hat{J}$ is defined by

$$
\hat{J}(A, B)=\frac{\partial}{\partial x} A \frac{\partial}{\partial Y} B-\frac{\partial}{\partial y} A \frac{\partial}{\partial X} B .
$$

Note that in A12, the PG-controlled ventilated thermocline strongly affects the QG evolution. It provides a background advection of QG PV as well as defines a background potential vorticity field (the term $\frac{f}{\frac{\partial}{\partial b} z_{o}}$ in A12) upon which the QG evolution occurs. Thus PG feedbacks on QG are strong, while feedbacks in the other direction do not exist. This implies that the system does not have a balanced energy budget.

The interior eddy Equation A12 admits baroclinically and barotropically unstable eddies, but the analysis to this point argues that the associated energy conversions are weak compared to those needed to obtain a global energy balance. To obtain a consistent energy budget, the dynamics behind the analysis must be enriched. In this paper, of the several possible choices for enriching the equations, we explore the consequences of a particular one, and then appeal to the analysis of a circulation model to support that choice as relevant.

Specifically, we connect the mesoscale and PG structure at leading order by an explicit introduction of anisotropy into the analysis. This is motivated by the recognition of strong boundary currents on the west sides of ocean basins. These currents have short spatial scales in the across-current direction and long spatial scales in the along-current direction, and are thus seen to fall somewhere between the primary scaling assumptions of the preceding multiple-scale analysis. Further, and importantly, they violate essential components of both QG and PG dynamics. First, the isopycnal depth anomalies occurring in the boundary jets are leading order on short scales, that is, isopycnals experience $O(1)$ depth changes over a deformation radius. In addition, in the cross-stream direction, the Rossby numbers of the flow naturally are found to have $O(1)$ values. Both characteristics are strongly non-QG and strongly non-PG.

Recognizing the appearance of the anisotropy is forced by the boundaries, we consider appending a boundary layer to the interior. As is well-known, boundary layer analyses involve rescaling the variables. If we consider the simplest case of a meridional boundary, then mass conservation requires the meridional flow be rescaled. Specifically, the interior southward mass flux scales like

$$
\text { Flux }=H V L_{\beta}=f_{o} R_{d}^{2} H,
$$

which requires a boundary layer flow of strength $f_{0} R_{d}$ if that mass is to be returned to the north over a width of the deformation radius. Thus the northward velocity in A3 must be rescaled by a factor of $\delta^{-1}\left(\hat{v}=\delta^{-1} v\right)$. The anisotropy required by the boundary is brought into the analysis by introducing a fast spatial scale into the zonal coordinate only, that is,

$$
\frac{\partial}{\partial X} \rightarrow \frac{\partial}{\partial X}+\frac{1}{\delta} \frac{\partial}{\partial x},
$$


while leaving the meridional derivatives untouched. Expanding the equations in $\delta$ as before yields the leading-order equations

$$
\begin{array}{r}
f \hat{v}_{o}=\frac{\partial}{\partial x} M_{o} \\
\frac{\partial}{\partial t} \hat{v}_{o}+u_{o} \frac{\partial}{\partial x} \hat{v}_{o}+\hat{v}_{o} \frac{\partial}{\partial Y} \hat{v}_{o}+f u_{o}=-\frac{\partial}{\partial Y} M_{o} \\
\frac{\partial}{\partial t} \frac{\partial}{\partial b} z_{o}+\frac{\partial}{\partial x}\left(u_{o} \frac{\partial}{\partial b} z_{o}\right)+\frac{\partial}{\partial Y}\left(\hat{v}_{o} \frac{\partial}{\partial b} z_{o}\right)=0 .
\end{array}
$$

Straightforward manipulations of A18 lead to

$$
\frac{\partial}{\partial t} \hat{q}+u_{o} \frac{\partial}{\partial x} \hat{q}+\hat{v}_{o} \frac{\partial}{\partial Y} \hat{q}=0
$$

where

$$
\hat{q}=\frac{\frac{\partial}{\partial x} \hat{v}_{o}+f}{\frac{\partial}{\partial b} z_{o}} .
$$

Equation A18 is recognized as conservation of potential vorticity by fluid parcels in the boundary layer, where boundary layer potential vorticity is given by A19. Exploiting hydrostatic dynamics, A19 can be rewritten as

$$
\frac{\partial}{\partial x} \frac{\partial}{\partial x} M_{o}+f \hat{q} \frac{\partial}{\partial b} \frac{\partial}{\partial b} M_{o}=f^{2}
$$

which should be recognized as an elliptic equation for $M_{0}$. Such equations, subject to conditions on the boundary and a far field value for $M_{o}$ set by the interior, can be inverted to give a unique answer. The simplicity and clarity of A20 provide one of the strongest motivations for conducting this analysis in buoyancy coordinates. Knowing $M_{o}$ determines $\hat{v}_{o}$ by geostrophy. Recalling that the interior leading-order thermocline is independent of rapid time $t$, solutions of A18 independent of $t$ are sought. Thus $u_{o}$ can be inferred from A18b and boundary layer mass flux can be expressed by a streamfunction

$$
\begin{aligned}
\frac{\partial}{\partial x} \psi & =\hat{v}_{o} \frac{\partial}{\partial b} z_{o} \\
-\frac{\partial}{\partial Y} \psi & =u_{o} \frac{\partial}{\partial b} z_{o}
\end{aligned}
$$

so $\hat{q}=\hat{q}(\psi)$. Equivalently, the boundary layer PV is determined by the interior PV. These equations essentially represent the addition of an inertial boundary layer to the ventilated thermocline.

This boundary layer analysis applies to currents along western ocean coasts. Such currents are not, however, confined to the coasts; rather, they separate from lateral topography and move into the ocean interior retaining their identity as a jet for considerable downstream distances. While there is some degree of anisotropy associated with such currents, numerical solutions and observations both show that the jet evolution is quite complex. It is not therefore clear if a reduced set of equations can be obtained that fully describe the leading-order behavior of separated jets. It appears that the full primitive equations are required, although the geographical location at which the jet source is located is known.

\section{Appendix B: The Connection Between Buoyancy Coordinates and Geopotential Coordinates and Regional Energetics}

We interpret the time-means $u, v, w$, and $p$ from the model output with the large-scale variables of the multiple-scale analysis, which is conducted in buoyancy coordinates. In general, time averaging in these two coordinate systems are not the same, but here the characteristics of the large-scale flow removes this issue, as we now show. 


\section{B1. Interior}

For the isotropic, ocean interior, the theoretical leading-order momentum equations are geostrophy, involving both large and small scales

$$
\begin{aligned}
& -f v_{o}=-\left.\frac{\partial}{\partial X}\right|_{b} M_{o}(X, Y, b)-\left.\frac{\partial}{\partial x}\right|_{b} M_{1}+\frac{\partial}{\partial z} \tau^{x} \\
& f u_{o}=-\left.\frac{\partial}{\partial Y}\right|_{b} M_{o}(X, Y, b)-\left.\frac{\partial}{\partial y}\right|_{b} M_{1}+\frac{\partial}{\partial z} \tau^{y},
\end{aligned}
$$

where the leading-order Montgomery potential, $M_{o}=p_{o}-b z_{o}$ is a function only of the long spatial scale coordinates, and the derivatives are taken along buoyancy surfaces, as indicated in this appendix by the notation $I_{b}$. We have also replaced the vertical turbulent stress with its form in geopotential coordinates, that is, we have used

$$
\frac{\frac{\partial}{\partial b} \tau^{x, y}}{\frac{\partial}{\partial b} z_{o}}=\frac{\partial}{\partial z} \tau^{x, y} .
$$

We have augmented geostrophy with vertical "turbulent" momentum transport, which is expected to be sizable only in the very near surface region where it conducts wind momentum into the fluid. Averaging this equation in space demonstrates that large-scale flow is geostrophic and independent of the mesoscale

$$
\begin{gathered}
f \overline{v_{o}}=\left.\frac{\partial}{\partial X}\right|_{b} M_{o}(X, Y, b)+\frac{\partial}{\partial z} \overline{\tau^{x}} \\
f \overline{u_{o}}=-\left.\frac{\partial}{\partial Y}\right|_{b} M_{o}(X, Y, b)+\frac{\partial}{\partial z} \overline{\tau^{y}},
\end{gathered}
$$

where the overbar $(\bar{x})$ denotes a spatial average. Expanding the Montgomery leads to

$$
\begin{aligned}
f \overline{v_{o}} & =\left.\frac{\partial}{\partial X}\right|_{b} p_{o}(X, Y, b)-\left.b \frac{\partial}{\partial X}\right|_{b} z_{o}+\frac{\partial}{\partial z} \overline{\tau^{x}} \\
-f \overline{u_{o}} & =-\left.\frac{\partial}{\partial Y}\right|_{b} p_{o}(X, Y, b)+\left.b \frac{\partial}{\partial Y}\right|_{b} z_{o}+\frac{\partial}{\partial z} \overline{\tau^{y}} .
\end{aligned}
$$

If we now define the function

$$
b_{o}\left(X, Y, z_{o}(X, Y, b), t\right)=b,
$$

the quantity $b$ in B4 can be replaced by $b_{o}$ and using the well-known formula for converting to geopotential coordinates from buoyancy coordinates

$$
\begin{aligned}
f \overline{v_{o}} & =\frac{\partial}{\partial X} p_{o}\left(X, Y, z_{o}\right)+\left.\frac{\partial}{\partial z} p_{o} \frac{\partial}{\partial X}\right|_{b} z_{o}-\left.b_{o} \frac{\partial}{\partial X}\right|_{b} z_{o}+\frac{\partial}{\partial z} \overline{\tau^{x}} \\
-f \overline{u_{o}} & =-\frac{\partial}{\partial Y} p_{o}\left(X, Y, z_{o}\right)-\left.\frac{\partial}{\partial z_{o}} p_{o} \frac{\partial}{\partial Y}\right|_{b} z_{o}+\left.b_{o} \frac{\partial}{\partial Y}\right|_{b} z_{o}+\frac{\partial}{\partial z} \overline{\tau^{y}} .
\end{aligned}
$$

With the hydrostatic equation

$$
\frac{\partial}{\partial z} p_{o}=b
$$

B4 reduces to geostrophy, augmented by turbulent momentum transport. 
In geopotential coordinates

$$
\begin{aligned}
f \overline{v_{o}} & =\frac{\partial}{\partial X} p_{o}(X, Y, z)+\frac{\partial}{\partial z} \overline{\tau^{x}} \\
-f \overline{u_{o}} & =-\frac{\partial}{\partial Y} p_{o}(X, Y, z)+\frac{\partial}{\partial z} \overline{\tau^{y}} .
\end{aligned}
$$

Vector multiplying by the large-scale horizontal velocities, which are the same in either coordinate system, leads to the interior energy equation B11, provided that

$$
\begin{aligned}
& \bar{u}_{o}=\bar{u}^{t} \\
& \bar{v}_{o}=\bar{v}^{t} \\
& \bar{p}_{o}=\bar{p}^{t}
\end{aligned}
$$

as we assumed.

\section{B2. Interior Energy}

Vector multiplying B8 by $\overline{\boldsymbol{u}}^{t}$ and using B9 yields

$$
0=-\nabla \cdot\left(\overline{\boldsymbol{u}}^{t} \bar{p}^{t}\right)+\bar{w}^{t} \bar{b}^{t}+\bar{u}^{t} \frac{\partial}{\partial z} \bar{\tau}^{t}+\bar{v}^{t} \frac{\partial}{\partial z} \bar{\tau}^{y}
$$

A volume integration of B10 gives

$$
\int_{S} \overline{\boldsymbol{u}}^{t}\left(\bar{p}^{t}+\bar{h}^{t}\right) d V=\int_{A} \overline{\boldsymbol{u}}^{t}(0) \cdot{\overline{\tau_{o}}}^{t} d A
$$

where interior viscous dissipation has been ignored, $\tau_{0}$ is the surface wind-stress, $\overline{\boldsymbol{u}}^{t}(0)$ is the surface velocity and $A$ is the ocean surface part of the surface $S$ bounding the volume $V$. We have also used the ventilated thermocline form of the tracer equations

$$
\overline{\boldsymbol{u}}^{t} \cdot \nabla \bar{\chi}^{t}=0
$$

where $\chi$ is a tracer. Importantly, eddy tracer transports are neglected in B12. The content of B11 reflects classical Sverdrup dynamics: the net export of energy from the domain is provided by wind forcing. Note the absence of kinetic energy flux divergence, consistent with the small Rossby number assumption behind geostrophy.

\section{B3. Coastal Jet}

For the anisotropic coastal jet, the momentum equations are A18, for convenience restated here in the appendix notation

$$
\begin{gathered}
f \hat{v}_{o}=\left.\frac{\partial}{\partial x}\right|_{b} M_{o} \\
\left.u_{o} \frac{\partial}{\partial x}\right|_{b} \hat{v}_{o}+\left.\hat{v}_{o} \frac{\partial}{\partial Y}\right|_{b} \hat{v}_{o}+f u_{o}=-\left.\frac{\partial}{\partial Y}\right|_{b} M_{o},
\end{gathered}
$$

where $u_{o}$ and $\hat{v}_{o}$ are independent of the rapid time scale. Equation B5 implies

$$
\hat{v}_{o}(x, Y, b, T)=\hat{v}_{o}\left(x, Y, b_{o}, T\right),
$$

with a similar equation holding for $u_{0}$. Converting the horizontal derivatives from buoyancy surfaces to geopotential surfaces leads to 


$$
\begin{gathered}
f \hat{v}_{o}=\frac{\partial}{\partial x} p_{o} \\
u_{o} \frac{\partial}{\partial x} \hat{v}_{o}+\hat{v}_{o} \frac{\partial}{\partial Y} \hat{v}_{o}+w_{o} \frac{\partial}{\partial z} \hat{v}_{o}+f u_{o}=-\frac{\partial}{\partial Y} p_{o}
\end{gathered}
$$

where the derivates are now taken on geopotential surfaces.

The coastal jet equations in geopotential coordinates are as follows:

$$
\begin{gathered}
f \bar{v}^{t}=\frac{\partial}{\partial x} \bar{p}^{t} \\
\bar{u}^{t} \frac{\partial}{\partial x} \bar{v}^{t}+\bar{v}^{t} \frac{\partial}{\partial y} \bar{v}^{t}+\bar{w}^{t} \frac{\partial}{\partial z} \bar{v}^{t}+f \bar{u}^{t}=-\frac{\partial}{\partial y} \bar{p}^{t} \\
\bar{v}^{t}-f \bar{u}^{t}=-\frac{\partial}{\partial y} \bar{p}^{t} \\
\frac{\partial}{\partial z} \bar{p}^{t}=\bar{b}^{t} \\
\nabla \cdot \overline{\boldsymbol{u}}^{t}=0 .
\end{gathered}
$$

Vector multiplying B16 by the time mean horizontal velocities leads to

$$
\nabla \cdot \overline{\boldsymbol{u}}^{t} \frac{\left(\bar{v}^{t}\right)^{2}}{2}=-\bar{u}^{t} \frac{\partial}{\partial x} \bar{p}^{t}-\bar{v}^{t} \frac{\partial}{\partial y} \bar{p}^{t}
$$

The absence of the rapid QG timescale from the governing equations implies mesoscale dynamics do not participate, as motivated by potential vorticity conservation A18. Integrating B17 over a coastal jet volume yields

$$
\int_{S} \overline{\boldsymbol{u}}^{t} \frac{\left(\bar{v}^{t}\right)^{2}}{2} \cdot \vec{n} d S=-\int_{V}\left[\bar{u}^{t} \frac{\partial}{\partial x} \bar{p}^{t}+\bar{v}^{t} \frac{\partial}{\partial y} \bar{p}^{t}\right] d V
$$

The interpretation of B17 is quite straight-forward: down or up pressure gradient flow will increase or decrease the kinetic energy flux. We will discuss this form of the energy equation for the coastal jet. If we include potential energy into B18, it becomes

$$
\int_{S} \overline{\boldsymbol{u}}^{t}\left(\frac{\left(\bar{v}^{t}\right)^{2}}{2}+\bar{p}^{t}+\bar{h}^{t}\right) \cdot \boldsymbol{n} d S=0
$$

That is, total energy is conserved, B12 having again been used.

\section{Appendix C: Mean and Eddy Energy With a Seawater Equation of State}

Here, we flesh out our full mean and eddy energy derivations. First, the time averaged hydrostatic balance is

$$
\frac{\partial}{\partial z} \bar{p}^{t}=\overline{b(\theta, S, P)}^{t}
$$

where the dependencies on potential temperature $\theta$, salinity, $S$, and static pressure $P=P_{o}-\rho_{o} g z$ explicitly appear. The quantity $P_{o}$ is a constant representing average atmospheric sea level pressure and $\rho_{o}$ is a Boussinesq reference density. We will approximate this balance using

$$
\overline{b(\theta, S, P)}^{t}=b\left(\bar{\theta}^{t}, \bar{S}^{t}, P\right),
$$

To see this, buoyancy at any one time can be related to the time mean thermodynamic quantities by Taylor expansion 
(a)

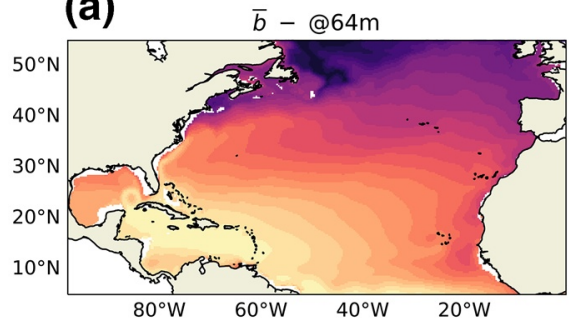

(c)

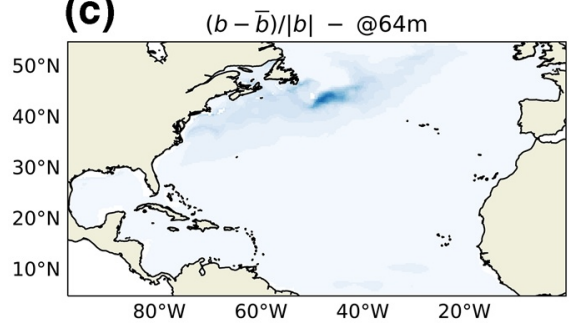

(b)

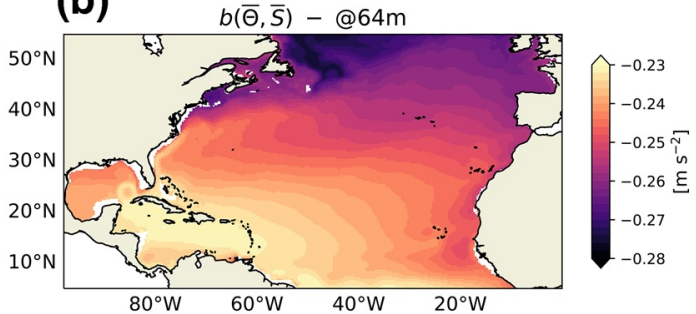

(d)

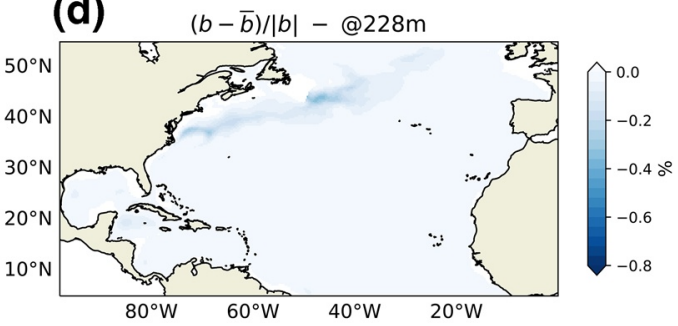

Figure C1. Comparison of time mean buoyancy to buoyancy computed using time mean $\theta$ and $S$. Panel (a) shows the mean buoyancy, and panel (b) the buoyancy based on mean tracers at a depth of $64 \mathrm{~m}$. The relative difference appears on panel (c), highlighting the small error $(<1 \%)$ made when computing buoyancy from time mean tracers. Panel (d) shows the relative difference but at $228 \mathrm{~m}$, where the error is even smaller.

$$
b(\theta, S, P)=b\left(\bar{\theta}^{t}+\theta^{\prime}, \bar{S}^{t}+S^{\prime}, P\right)+b_{\theta}\left(\bar{\theta}^{t}\right) \theta^{\prime}+b_{S}\left(\bar{S}^{t}\right) S^{\prime}+O\left(\theta^{\prime 2}, S^{\prime 2}, \theta^{\prime} S^{\prime}\right) .
$$

The first-order contributions vanish upon time averaging, so to second order we recover $\mathrm{C} 2$. Comparisons between $\overline{b(\theta, S, P)}^{t}$ and $b\left(\bar{\theta}^{t}, \bar{S}^{t}, P\right)$ in Figure $\mathrm{C} 1$ show that $\mathrm{C} 3$ and $\mathrm{C} 2$ are good approximations.

Adding and subtracting $\bar{w}^{t} \bar{b}^{t}$, in view of $\mathrm{C} 1$, converts 1 to

$$
\left.\frac{\partial}{\partial x_{i}}\left(\bar{u}_{i}^{t} \hat{K}\right)=-\frac{\partial}{\partial x_{i}}\left(\bar{u}_{i}^{t} \bar{p}^{t}\right)-\bar{\epsilon}^{t}+\frac{\partial}{\partial x_{i}}\left(v \frac{\partial}{\partial x_{i}} \hat{K}\right)-{\overline{u_{I}}}^{t} \frac{\partial}{\partial x_{i}} \overline{\left(\bar{u}_{i}^{\prime} u_{I}^{t}\right.}\right)+\bar{w}^{t} \bar{b}^{t}
$$

Following Young (2010), introducing potential enthalpy,

$$
h(\theta, S, P)=\int_{P_{o}}^{P} \frac{b}{g \rho_{o}} d P
$$

where the integral assumes fixed potential temperature and salinity. Thus we can define the mean potential enthalpy, following Young (2010)

$$
\bar{h}^{t}=\int_{P_{o}}^{P} \frac{\bar{b}^{t}}{g \rho_{o}} d P
$$

and its mean advective derivative

$$
\overline{\boldsymbol{u}}^{t} \cdot \nabla \bar{h}^{t}=-\bar{w}^{t} \bar{b}^{t}+{\overline{h_{\theta}}}^{t} \overline{\boldsymbol{u}}^{t} \cdot \nabla \bar{\theta}^{t}+{\overline{h_{S}}}^{t} \overline{\boldsymbol{u}}^{t} \cdot \nabla \bar{S}^{t}
$$

The mean tracer equations are as follows:

$$
\overline{\boldsymbol{u}}^{t} \cdot \nabla \bar{\chi}^{t}=-\nabla \cdot \overline{\boldsymbol{u}}^{\prime} \chi^{t},
$$

where $\chi$ is either $\theta$ or $S$, and nonhydrostatic and diffusive processes have been ignored. The tracer derivatives of mean potential enthalpy can be written 


$$
\bar{h}_{\chi}^{t}=\int_{P_{o}}^{P} \frac{\bar{b}_{\chi}^{t}}{g \rho_{o}} d P .
$$

From $\mathrm{C} 7$ and $\mathrm{C} 8$, it follows that

$$
\nabla \cdot \overline{\boldsymbol{u}}^{t} \bar{h}^{t}=-\bar{w}^{t} \bar{b}^{t}-\overline{w^{\prime} b^{\prime}}-\nabla \cdot\left(\overline{\boldsymbol{u}^{\prime} \theta^{\prime}} \int_{P_{o}}^{P} \frac{\bar{b}_{\theta}^{t}}{g \rho_{o}} d P+\overline{\boldsymbol{u}^{\prime} S^{t}} \int_{P_{o}}^{P} \frac{\bar{b}_{S}^{t}}{g \rho_{o}} d P\right)
$$

where

$$
\overline{w^{\prime} b^{\prime}}=\bar{b}_{\theta}^{t} \overline{w^{\prime} \theta^{\prime}}+\bar{b}_{S}^{t} \overline{w^{\prime} S^{\prime}}
$$

has been used and second-order tracer derivatives of mean potential enthalpy (e.g., $\bar{h}_{\theta S}^{t}$ ) have been ignored. The exact potential energy equation is

$$
\frac{d}{d t} h=-w b+h_{\theta} \frac{d}{d t} \theta+h_{S} \frac{d}{d t} S
$$

so upon time averaging

$$
\nabla \cdot \overline{\boldsymbol{u h}}^{t}=-\overline{w b}^{t}
$$

to the neglect of nonhydrostatic and diffusive processes. Equation $\mathrm{C} 4$ can then be written as 3 .

\section{Appendix D: Diagnosing Kinetic Energy From the MITgem}

We outline here the energy diagnostic method we developed which is robust given the many design parameters involved in the MITgcm. Our method relies heavily on diagnostics traditionally available from the MITgcm. For example, the zonal momentum equation can be written as

$$
\frac{\partial}{\partial t} u=-\nabla \cdot u u+f v-\frac{\partial}{\partial x} p+D^{u}
$$

where $u$ is the zonal velocity, $\boldsymbol{u}$ is the three dimensional velocity, $f$ the Coriolis parameter, $v$ the meridional velocity, $p$ the pressure, and $D^{u}$ the zonal viscous operator. The MITgcm provides fields of the partial time derivative of $u$, the combination of the advective terms and the Coriolis acceleration, the pressure gradient and the contributions to the viscous dissipation (there are several). Similar fields can be output for the meridional equation. The kinetic energy equation is developed analytically by multiplying D1 by the zonal velocity, performing a similar procedure to the meridional equation and adding them. The MITgcm uses an Adams-Bashforth explicit time stepping for momentum, such that the tendency terms on the righthand side of D1) are evaluated at mid-point in time. They are thus multiplied by their associated velocities $u^{n+1 / 2}=\frac{1}{2}\left(u^{n}+u^{n+1}\right)$ to insure that the time integrated kinetic energy trends are equal to

$$
\int_{t_{i}}^{t_{f}} \partial_{t} K d t=K\left(t_{f}\right)-K\left(t_{i}\right) .
$$

We follow this recipe during model execution using the model momentum diagnostics. A numerical issue is that the MITgcm employs a C grid (Marshall et al., 1997), so that the locations in space of $u, v$ and the various tracers differ. The diagnostic fields balance the budgets of the various quantities at the native grid locations of the variables, for example, the momentum budget of the zonal velocity is balanced at the zonal velocity grid points, etc. We have opted to balance the energy budget at the tracer points, according to 


$$
\nabla \cdot \boldsymbol{u} K=\overline{u(\nabla \cdot \boldsymbol{u} u-f v)}^{i}+\overline{v(\nabla \cdot \boldsymbol{u} v+f u)}^{j}
$$

where the overbars $-^{i}$ and $-^{j}$ denote the average of the zonal and meridional momentum neighbors of a given tracer point along the $x$ - and $y$-axis, respectively. Following a similar procedure for each of the contributors to the momentum equations results in a machine precision kinetic energy balance.

\section{Data Availability Statement}

The contributions to the mean kinetic energy equation from our model run are available at http://ocean.fsu. edu/ qjamet/share/data/energetics/.

\section{Acknowledgments}

This work was supported through NSF grants OCE-1434780, OCE-1537304, OCE-1829856, and OCE-1941963 and the French "Make Our Planet Great Again" program managed by the Agence Nationale de la Recherche under the Programme d'Investissement d'Avenir, with the reference ANR-18MPGA-0002. We would like to thank the editor Stephen Griffies and three anonymous reviewers for their careful reviews, which have contributed significantly to the final presentation. The authors would also like to acknowledge many interesting conversations with Prof. Ian Grooms. W. K. Dewar in particular would like to recognize the formative influence that N. P. Fofonoff had on him. N. P. Fofonoff was a kind man, and his quiet way belied an enormous intellect that is greatly missed.

\section{References}

Bleck, R., \& Chassignet, E. (1994). Simulating the oceanic circulation with isopycnic coordinate models. The Pennsylvania Academy of Science.

Chassignet, E., \& Xu, X. (2017). Impact of horizontal resolution (1/12 to 1/50) on Gulf Stream separation, penetration, and variability. Journal of Physical Oceanography, 47, 1999-2021.

Deremble, B., Dewar, W., \& Wienders, N. (2014). Potential vorticity budgets in the North Atlantic Ocean. Journal of Physical Oceanography, 44, 164-178.

Deremble, B., Wienders, N., \& Dewar, W. (2013). CheapAML: A simple, atmospheric boundary layer model for use in ocean-only model calculations. Monthly Weather Review, 141, 809-821.

Fairall, C., Bradley, E., Hare, J., Grachev, A., \& Edson, J. (2003). Bulk parameterization of air-sea fluxes: Updates and verification for the COARE algorithm. Journal of Climate, 16, 571-591.

Ferrari, R., \& Wunsch, C. (2009). Ocean circulation kinetic energy - Reservoirs, sources and sinks. Annual Review of Fluid Mechanics, 41, $253-282$.

Fofonoff, N. (1981). The gulf stream system. Evolution of physical oceanography. MIT Press.

Gent, P., \& McWilliams, J. (1990). Isopycnal mixing in ocean circulation models. Journal of Physical Oceanography, 20, $150-155$.

Gill, A., Green, J., \& Simmons, A. (1974). Energy partition in the large-scale ocean circulation and the production of mid-ocean eddies. Deep Sea Research and Oceanographic Abstracts, 21, 499-528.

Greatbatch, R., Zhai, X., Claus, M., Czeschel, L., \& Rath, W. (2010). Transport driven by eddy momentum fluxes in the Gulf Stream Extension region. Geophysical Research Letters, 37, L24401. https://doi.org/10.1029/2010GL045473

Grooms, I., Julien, K., \& Fox-Kemper, B. (2011). On the interactions between planetary geostrophy and mesoscale eddies. Dynamics of Atmospheres and Oceans, 51, 109-136.

Harrison, D., \& Robinson, A. (1978). Energy analysis of open regions of turbulent flows - Mean eddy energetics of a numerical ocean circulation experiment. Dynamics of Atmospheres and Oceans, 2, 185-211.

Holland, W. (1978). The role of mesoscale eddies in the general circulation of the ocean - numerical experiments using a wind-driven quasi-geostrophic model. Journal of Physical Oceanography, 8, 363-392.

Holland, W., \& Lin, L. (1975). On the generation of mesoscale eddies and their contribution to the oceanic general circulation. I. A preliminary numerical experiment. Journal of Physical Oceanography, 5, 642-657.

Hughes, C., \& de Cuevas, B. (2001). Why western boundary currents in realistic oceans are inviscid: A link between form stress and bottom pressure torque. Journal of Physical Oceanography, 31, 2871-2885.

Jackett, D., \& McDougall, T. (1995). Minimal adjustment of hydrographic profiles to achieve static stability. Journal of Atmospheric and Oceanic Technology, 12, 381-389.

Jamet, Q., Dewar, W., Wienders, N., \& Deremble, B. (2019). Spatio-temporal patterns of chaos in the Atlantic Overturning Circulation. Geophysical Research Letters, 46, 7509-7517. https://doi.org/10.1029/2019GL082552

Kang, D., \& Curchitser, E. (2015). Energetics of eddy-mean flow interactions in the Gulf Stream region. Journal of Physical Oceanography, $45,1103-1120$.

Luyten, J., Pedlosky, J., \& Stommel, H. (1983). The ventilated thermocline. Journal of Physical Oceanography, 13, $292-309$.

Marshall, J., Hill, C., Perelman, L., \& Adcroft, A. (1997). Hydrostatic, quasi-hydrostatic and non-hydrostatic ocean modeling. Journal of Geophysical Research, 102, 5733-5753.

Molines, J., Barnier, B., Penduff, T., Treguier, A., \& Le Sommer, J. (2014). ORCA 12. L46 climatological and interannual simulations forced with DFS4.4: GJM02 and MJM88 (Technical Report No. DRAKKAR-2014-03-19) DRAKKAR Group Experiment. Retrieved from http:// www.Drakkar-ocean.eu

Montgomery, R. (1937). A suggested method for representing gradient flow in isentropic surfaces. Bulletin of the American Meteorological Society, 18, 210-212.

Pedlosky, J. (1984). The equations for geostrophic motion in the ocean. Journal of Physical Oceanography, 14, 15-30.

Plumb, R., \& Ferrari, R. (2005). Transformed Eulerian-mean theory. Part I: Nonquasigeostrophic theory for eddies on a zonal-mean flow. Journal of Physical Oceanography, 35, 165-174.

Rhines, P., \& Young, W. (1982). A theory of wind-driven circulation. I. Mid-ocean gyres. Journal of Marine Research, 40, 559-596.

Robinson, A., Harrison, D., Maint, Y., \& Semtner, A. (1977). Eddies and the general circulation of an idealized oceanic gyre: A wind and thermally driven primitive equation numerical experiment. Journal of Physical Oceanography, 7, 182-207.

Schoonover, J., Dewar, W., Wienders, N., Gula, J., McWilliams, J., Molemaker, J., et al. (2016). North Atlantic barotropic vorticity balances in numerical models. Journal of Physical Oceanography, 46, 289-303.

Semtner, A., \& Mintz, Y. (1977). Numerical simulation of the Gulf Stream and mid-ocean eddies. Journal of Physical Oceanography, 7 , 208-230. 
Serazin, G., Penduff, T., Gregorio, S., Barnier, B., Molines, J., \& Terray, L. (2015). Intrinsic variability of sea level from global ocean simulations: Spatiotemporal scales. Journal of Climate, 10, 4279-4292.

St Laurent, L., \& Simmons, H. (2006). Estimates of power consumed by mixing in the ocean interior. Journal of Climate, 19, 4877-4890 Welander, P. (1959). An advective model of the ocean thermocline. Tellus, 11, 309-318.

Worthington, L. (1976). On the North Atlantic circulation. Baltimore, MD: Johns Hopkins University Press.

Wunsch, C. (1998). The work done by the wind on the oceanic general circulation. Journal of Physical Oceanography, 28, 2331-2339.

Wunsch, C., \& Ferrari, R. (2004). Vertical mixing, energy, and the general circulation of the oceans. Annual Review of Fluid Mechanics, 36 , 281-314. https://doi.org/10.1146/annurev.fluid.36.050802.122121

Young, W. (2010). Dynamic enthalpy, conservative temperature and the seawater Boussinesq approximation. Journal of Physical Oceanography, 40, 394-400.

Young, W. (2012). An exact thickness-weighted average formulation of the Boussinesq equations. Journal of Physical Oceanography, 42, 692-707.

Zhai, X., Johnson, H., Marshall, D., \& Wunsch, C. (2012). On the wind power input to the ocean general circulation. Journal of Physical Oceanography, 42, 1357-1365.

Zhai, X., \& Marshall, D. (2013). Vertical eddy energy fluxes in the North Atlantic subtropical and subpolar gyres. Journal of Physical Oceanography, 43, 95-103. 